\title{
Large-scale two-dimensional turbulence in the atmosphere
}

\author{
Article \\ Published Version
}

Boer, G. J. and Shepherd, T. G. (1983) Large-scale twodimensional turbulence in the atmosphere. Journal of the Atmospheric Sciences, 40 (1). pp. 164-184. ISSN 1520-0469 doi: https://doi.org/10.1175/15200469(1983)040<0164:LSTDTI>2.0.CO;2 Available at https://centaur.reading.ac.uk/32999/

It is advisable to refer to the publisher's version if you intend to cite from the work. See Guidance on citing.

Published version at: http://dx.doi.org/10.1175/1520-0469(1983)040<0164:LSTDTI>2.0.CO;2

To link to this article DOI: http://dx.doi.org/10.1175/1520-

0469(1983)040<0164:LSTDTI>2.0.CO;2

Publisher: American Meteorological Society

All outputs in CentAUR are protected by Intellectual Property Rights law, including copyright law. Copyright and IPR is retained by the creators or other copyright holders. Terms and conditions for use of this material are defined in the End User Agreement.

\section{www.reading.ac.uk/centaur}

\section{CentAUR}


Central Archive at the University of Reading

Reading's research outputs online 


\title{
Large-Scale Two-Dimensional Turbulence in the Atmosphere
}

\author{
G. J. BOER AND T. G. SHEPHERD \\ Canadian Climate Centre, Downsview, Ontario M3H $5 T 4$ Canada
}

(Manuscript received 14 May 1982, in final form 13 September 1982)

\begin{abstract}
Global FGGE data are used to investigate several aspects of large-scale turbulence in the atmosphere. The approach follows that for two-dimensional, nondivergent turbulent flows which are homogeneous and isotropic on the sphere. Spectra of kinetic energy, enstrophy and available potential energy are obtained for both the stationary and transient parts of the flow. Nonlinear interaction terms and fluxes of energy and enstrophy through wavenumber space are calculated and compared with the theory. A possible method of parameterizing the interactions with unresolved scales is considered.

Two rather different flow regimes are found in wavenumber space. The high-wavenumber regime is dominated by the transient components of the flow and exhibits, at least approximately, several of the conditions characterizing homogeneous and isotropic turbulence. This region of wavenumber space also displays some of the features of an enstrophy-cascading inertial subrange. The low-wavenumber region, on the other hand, is dominated by the stationary component of the flow, exhibits marked anisotropy and, in contrast to the high-wavenumber regime, displays a marked change between January and July.
\end{abstract}

\section{Introduction}

The striking differences between two- and threedimensional turbulent flows in Cartesian geometry have been discussed extensively in the literature (Fjørtoft, 1953; Kraichnan, 1967; Batchelor, 1969; Leith, 1971; Tennekes, 1978). Although both phenomena exhibit the properties of randomness and nonperiodicity due to nonlinear interactions, two-dimensional turbulence is characterized by a tendency for "upscale" transfer of kinetic energy, i.e., from smaller to larger scales, while three-dimensional turbulence is characterized by "down-scale" energy transfer. The so-called "negative viscosity" effect of two-dimensional turbulence is a consequence of enstrophy conservation in a two-dimensional fluid which prevents an energy cascade to smaller scales via vortex stretching.

Two-dimensional homogeneous isotropic turbulence might seem to be of rather academic interest in the study of the large-scale behavior of the atmosphere. The atmosphere, however, seems to exhibit at least some of the features which characterize turbulent flows of this kind. This partial correspondence may be made plausible by arguing that the very large ratio of horizontal to vertical scale in the atmosphere means that atmospheric flows are at least quasi-twodimensional and certainly eliminates the possibility of three-dimensional homogeneous isotropic turbulence, except at the smaller scales of motion.

A more sophisticated approach is that of quasigeostrophic turbulence developed by Charney (1971). In this theory, the quasi-two-dimensional nature of the atmosphere as implied by the quasi-geostrophic constraint is used to scale the vertical coordinate and to develop a theory of three-dimensional isotropic turbulence in the scaled coordinates.

In what follows, some of the basic results for twodimensional plane turbulence and for quasi-geostrophic turbulence are reviewed, the corresponding results in terms of the spherical harmonic two-dimensional wavenumber $n$ for flow on a sphere are obtained and global atmospheric data for the months of January and July 1979 are analyzed in the light of these ideas.

\section{Two-dimensional plane turbulent flow}

\section{a. Equilibrium theory}

In both two- and three-dimensional turbulent flow the equilibrium theory of Kolmogorov (Kolmogorov, 1941; Batchelor, 1953) may be considered for inertial ranges. Two particularly simple turbulence solutions for nondivergent barotropic flow on a plane are characterized by energy-cascading and enstrophy-cascading inertial subranges (Kraichnan, 1967; Leith, 1968). In the first of these, a constant negative (i.e., upscale) flux of energy is accompanied by a vanishing flux of enstrophy through a wavenumber range where the energy spectrum follows a $-5 / 3$ power law. In the second case, a constant positive flux of enstrophy and a vanishing flux of energy are associated with an energy spectrum which follows a -3 power law.

A model which is thought to have some resemblance to the atmosphere postulates an energy-en- 
strophy source localized in a certain wavenumber range, with the removal of energy at large scales and the removal of enstrophy at small scales. The source and sink regions are connected by two inertial subranges, respectively transporting energy and enstrophy to their sink regions. Lilly (1972) has produced this behavior in a numerical model.

Attention has focussed primarily on the enstrophycascading subrange as a possible mechanism in largescale atmospheric turbulence. Observational studies (Baer, 1972; Chen and Wiin-Nielsen, 1978) have suggested that such a subrange might exist beyond the baroclinic excitation scales.

Turbulent flows characterized by inertial subranges are, of course, rather special cases. More general turbulent flows involving sources and sinks of energy and enstrophy at many scales are hardly implausible in the real atmosphere. There is some indirect evidence (Leith, 1971) to suggest excitation at wavelengths smaller than those expected from classical baroclinic instability theories. Observational studies such as those by Kung and co-workers (e.g., Kung and Baker, 1975) may also be interpreted to support such a possibility. The prospect that such sources may extend past the resolution limits of synoptic-scale observational networks and current general circulation and numerical models has important ramifications for understanding and modelling atmospheric flow.

\section{b. Quasi-geostrophic theory}

Charney (1971) has argued that the conservation of "pseudopotential vorticity" in three-dimensional quasi-geostrophic flow is analogous to the conservation of enstrophy in two-dimensional inviscid flow. The kinetic energy and available potential energy equations combined give a total energy equation which is analogous to the kinetic energy equation in two dimensions. An inertial subrange is then possible between a source region and a high-wavenumber enstrophy sink region.

These results are obtained under a number of assumptions, including that of isotropy with respect to the horizontal and scaled vertical coordinates. This in turn leads to the prediction of energy equipartition between the two components of kinetic energy and the available potential energy. In this system the enstrophy-cascading subrange exhibits a -3 power law for kinetic energy and available potential energy.

There are several difficulties in attempting to verify these predictions from observations. The interpretation of global data in terms of quasi-geostrophic formulas may not be everywhere applicable. The isotropy with respect to the horizontal and scaled vertical coordinates suggests that the energy distribution should be investigated in terms of a three-dimensional spectral index. The choice of the vertical represen- tation is not obvious and has an effect on the results, as discussed for instance by Baer $(1974,1981)$.

\section{c. Application to observed data}

In the face of inhomogeneity, anisotropy, vertical motions, and source-sink terms distributed throughout the spectral domain, how can the real atmosphere be expected to behave like a simple two-dimensional turbulent fluid? The answer is that while not in precise agreement with the idealized system, the constraints on atmospheric motion may nevertheless lead to qualitative behavior which is similar to that predicted by simple theory so that the physical mechanisms of two-dimensional turbulence may play a role in atmospheric dynamics:

The approach adopted here is to analyze meteorological observations in a manner consistent with two-dimensional theory, and to an extent with quasigeostrophic theory, in an attempt to clarify the degree to which large-scale atmospheric turbulence resembles that of simple turbulence theory.

\section{Two-dimensional Cartesian turbulence}

\section{a. Homogeneous and isotropic turbulence}

Homogeneous and isotropic turbulence is characterized by statistics which are independent of the location and orientation of the coordinate axes. Turbulence statistics are therefore expressed (Leith, 1967; $1968,1971)$ as functions of a single-scale parameter, namely the wavenumber $k=\left(k_{x}^{2}+k_{y}^{2}\right)^{1 / 2}$. For twodimensional, planar, homogeneous, isotropic, nondivergent turbulent flows, where $\mathbf{V}=\mathbf{k} \times \nabla \psi$ and $\zeta=\mathbf{k} \cdot \boldsymbol{\nabla} \times \mathbf{V}=\nabla^{2} \psi$, it follows that

$$
\begin{aligned}
1 / 2 \overline{\mathbf{V} \cdot \mathbf{V}} & =\int E(k) d k \\
1 / 2 \overline{\zeta^{2}} & =\int G(k) d k \\
\overline{u^{2}} & =\overline{v^{2}}=\int E(k) d k \\
\overline{u v} & =0 \\
E(k) & =\pi k^{3} \overline{|\psi(k)|^{2}} \\
G(k) & =k^{2} E(k)
\end{aligned},
$$

where the overbar represents a probability average. In other words, the energy and enstrophy spectra depend only on the two-dimensional wavenumber $k$ as does the streamfunction. Energy and enstrophy are simply related, there is an equal partition of energy between the two velocity components, and the velocity components are uncorrelated. 


\section{b. Energy and enstrophy equations}

The spectral kinetic energy equation for two-dimensional, homogeneous isotropic turbulence may be written as

$$
\frac{\partial E(k)}{\partial t}=I(k)+2\left[\alpha(k)-\nu k^{2}\right] E(k)
$$

where $I(k)$ is the nonlinear transfer function representing the interchange of energy at wavenumber $k$ with all other wavenumbers, $\alpha(k)$ is a (presumed) isotropic forcing term, and $\nu$ is the kinematic viscosity. The associated spectral enstrophy equation is

$$
\frac{\partial G(k)}{\partial t}=J(k)+2\left[\alpha(k)-\nu k^{2}\right] G(k)
$$

The inertial transfer terms $I$ and $J$ serve only to redistribute energy and enstrophy among the various wavenumbers, not to change the total amount. This property allows the definition of energy and enstrophy flux functions $F$ and $H$, respectively, as

$$
\left.\begin{array}{l}
I=-\frac{\partial F}{\partial k} \\
J=-\frac{\partial H}{\partial k}
\end{array}\right\},
$$

where it follows that

$$
\frac{\partial H}{\partial k}=k^{2} \frac{\partial F}{\partial k} .
$$

The requirement that

$$
0=\int_{0}^{\infty} I d k=\int_{0}^{\infty} J d k
$$

implies that the flux functions $F$ and $H$ vanish at sufficiently large $k$. It follows also that $F$ must satisfy the constraint

$$
0=\int_{0}^{\infty} \frac{\partial H}{\partial k} d k=\int_{0}^{\infty} k^{2} \frac{\partial F}{\partial k} d k=-2 \int_{0}^{\infty} k F d k
$$

In an inertial subrange

$$
\frac{\partial E}{\partial t}=0=2\left(\alpha-\nu k^{2}\right) E
$$

so that $I=-\partial F / \partial k=0$ and similarly $J=-\partial H / \partial k$ $=0$. Such a subrange is characterized by vanishing interaction terms and constant fluxes of energy and enstrophy. Assuming that within such a subrange the energy spectrum is determined solely by the wavenumber and the flux of energy or enstrophy, dimensional arguments lead to the possible existence of two classes of subranges characterized by

and

$$
\left.\begin{array}{ll}
E \propto \varepsilon^{2 / 3} k^{-5 / 3}, & F=-\varepsilon, H=0 \\
E \propto \eta^{2 / 3} k^{-3}, & F=0, H=\eta
\end{array}\right\} .
$$

The first of these is the energy-cascading subrange with $-5 / 3$ spectral power law, negative energy flux of magnitude $\varepsilon$ and zero enstrophy flux. The second subrange is the enstrophy-cascading subrange with -3 spectral power law, downscale enstrophy flux of magnitude $\eta$ and zero energy flux.

\section{c. Parameterization of unresolved scales}

Both observational studies of atmospheric behavior and attempts to model large-scale atmospheric flow, using general circulation and forecast models, are made difficult by the finite resolution of the observations and of the models. One reason for studying the turbulent behavior of the atmosphere is to gain the knowledge required to develop parameterizations of the effects of unresolved scales on the scales explicitly carried in numerical models.

If it is assumed that the data from the atmosphere or the numerical model is truncated at some wavenumber $k_{*}$, the energy equation corresponding to (2) is written as

$$
\begin{aligned}
\frac{\partial E(k)}{\partial t}=I^{R}(k) & +I^{U}(k) \\
& +2\left[\alpha(k)-\nu k^{2}\right] E(k), \text { for } k \leqslant k_{*},
\end{aligned}
$$

where $I(k)=I^{R}(k)+I^{U}(k)$ has been split into a term involving interactions with waves explicitly resolved, that is for which $k \leqslant k_{*}$, and a second term $I^{U}(k)$ representing interactions with waves beyond the truncation limit. Eq. (7) will be correct for $k \leqslant k_{*}$ and expressible in terms of the resolved scales only if a suitable parameterized expression for $I^{U}(k)$ in terms of the resolved scales can be found.

One approach suggested by Leith (1971) is to assume that the truncation wavenumber falls in the enstrophy cascading subrange where

$$
0=\frac{\partial E(k)}{\partial t}=I^{R}(k)+I^{U}(k)=2\left[\alpha(k)-\nu k^{2}\right] E(k) .
$$

Then

$$
I^{U}(k)=-I^{R}(k)
$$

and if the resolved transfer rate is known, the effect of the unresolved scales is just that necessary to make the sum vanish as required in this subrange.

This relationship is formulated by Leith as an effective viscosity in the form

$$
I^{U}(k)=2 \nu_{*} k^{2} E(k)=-I^{R}(k),
$$

so that

$$
\nu_{*}(k)=\frac{-I^{R}(k)}{2 k^{2} E(k)} .
$$

The form of the unresolved interaction term is taken as

$$
I^{U}(k)=-I^{R}(k) \equiv L(k)=-2 \eta^{1 / 3} f\left(k / k_{*}\right) E(k),
$$


which is dimensionally consistent and depends on the constant enstrophy cascade rate $\eta$. The dimensionless function $f\left(k / k_{*}\right)$ must be calculated in some manner.

In his paper, Leith (1971) calculated such a function, based on a presumed enstrophy-cascading inertial subrange where the energy spectrum follows a -3 power law, by evaluating the resolved interaction terms $I^{R}(k)$ using the "eddy-damped Markovian approximation" and evaluating $f$ from the expression (9). This function may be estimated from atmospheric data under various assumptions.

\section{Two-dimensional turbulence in terms of spherical harmonics}

\section{a. Spherical harmonic representation}

For large-scale turbulence in the atmosphere the assumption of Cartesian geometry used in the previous section is no longer appropriate. The analogy between Fourier decomposition on a plane and spherical harmonic decomposition of turbulent flow on a sphere has been pointed out by Baer (1972) and WiinNielsen (1972) among others. The characteristics of homogeneous and isotropic turbulence on the sphere are worked out in the accompanying paper (Boer, 1983), hereinafter referred to as B. In particular, spectral and covariance relationships analogous to (1) are obtained where the scale index $n$ replaces the wavenumber $k$.

For application to atmospheric data where homogeneity and isotropy cannot be assumed $a$ priori, calculation of spectral quantities proceeds by averaging in the horizontal and in time. In such calculations the time average replaces the probability average used in B. The two are equivalent only under conditions of stationarity and homogeneity.

For calculations from data, where the horizontal average is represented by angular braces and the time average by an overbar,

$$
\begin{aligned}
\left\langle\overline{1 / 2 \zeta^{2}}\right\rangle & =\sum_{\alpha} G_{\alpha}=1 / 4 \sum_{\alpha} \overline{\left|\zeta_{\alpha}\right|^{2}}=\frac{1}{4} \sum_{\alpha} \frac{n^{2}(n+1)^{2}}{a^{4}} \mid \overline{\left.\psi_{\alpha}\right|^{2}} \\
& =\sum_{n=0}^{N} G_{n},
\end{aligned}
$$

where

$$
G_{n}=\sum_{m=-n}^{n} G_{\alpha}=\frac{1}{4} \sum_{m=-n}^{n} \frac{n^{2}(n+1)^{2}}{a^{4}} \overline{\left|\psi_{n}^{m}\right|^{2}}
$$

is the contribution to the enstrophy associated with the two-dimensional scale $n$. Note that the radius of the earth $a$ is carried explicitly in these calculations.

The kinetic energy is written as

$$
\begin{aligned}
\langle 1 / 2 \mathbf{V} \cdot \mathbf{V}\rangle & =\left\langle\overline{\nabla \cdot(\psi \nabla \psi)-\psi \nabla^{2} \psi}\right\rangle=\sum_{\alpha} E_{\alpha} \\
& =\frac{1}{4} \sum_{\alpha} \frac{n(n+1)}{a^{2}} \overline{\left|\psi_{\alpha}\right|^{2}}=\frac{1}{4} \sum_{\alpha} \frac{a^{2}}{n(n+1)} \overline{\left|\zeta_{\alpha}\right|^{2}} \\
& =\sum_{n=0}^{N} E_{n},
\end{aligned}
$$

where

$$
\left.\begin{array}{rl}
E_{n}=\sum_{m=-n}^{n} E_{\alpha} & =\frac{1}{4} \sum_{m=-n}^{n} \frac{n(n+1)}{a^{2}} \overline{\left|\psi_{n}^{m}\right|^{2}} \\
G_{n} & =\frac{n(n+1)}{a^{2}} E_{n}
\end{array}\right\} .
$$

The contribution to the available potential energy at a pressure level is given by

$$
\begin{aligned}
A & =\left\langle 1 / 2 \overline{C_{p} \gamma(T-\langle T\rangle)^{2}}\right\rangle=\sum_{\alpha} A_{\alpha} \\
& =\sum_{n=1}^{N} A_{n},
\end{aligned}
$$

where

$$
\left.\begin{array}{c}
A_{n}=1 / 4 \sum_{m=-n}^{n} C_{p} \gamma \overline{\left.T_{n}^{m}\right|^{2}} \\
\gamma=\frac{-\kappa}{p}\left(\frac{\theta}{T}\right)\left(\frac{\partial\langle\theta\rangle}{\partial p}\right)^{-1}
\end{array}\right\}
$$

\section{b. Equipartition}

As shown in B (Section 4a), the condition of homogeneity and isotropy of the streamfunction field on the sphere implies that energy and enstropy spectra are functions only of scalar wavenumber $n$, that kinetic energy is partitioned equally between velocity components, and that the velocity components are uncorrelated.

In this case, $\overline{\left|\psi_{n}^{m}\right|^{2}}$ is independent of $m$ and the spectra of the streamfunction, energy and enstropy are

$$
\begin{aligned}
& D_{n}=\sum_{m=-n}^{n} 1 / 2 \overline{\left|\psi_{n}^{m}\right|^{2}}=[(2 n+1) / 2] \overline{\left|\psi_{n}^{(m)}\right|^{2}}, \\
& E_{n}=\frac{n(n+1)}{2 a^{2}} D_{n}, \\
& G_{n}=\frac{n(n+1)}{a^{2}} E_{n} .
\end{aligned}
$$

Although it is shown in B that homogeneity and isotropy of the streamfunction implies equipartition of energy between velocity components in the sense that 


$$
\overline{u^{2}}=\overline{v^{2}}=1 / 2\left(\overline{u^{2}}+\overline{v^{2}}\right)=E=\sum_{n} E_{n},
$$

it is not clear how to express the spectra of the individual velocity components from data in order to test this relationship.

Baer (1972) calculated the spectrum of kinetic energy by expanding the zonal and meridional velocity components $u, v$ in terms of spherical harmonics

$$
(u, v)=\sum_{\alpha}\left(u_{\alpha}, v_{\alpha}\right) Y_{\alpha} .
$$

For this decomposition

$$
\begin{aligned}
1 / 2\left\langle\overline{u^{2}}\right\rangle & =\sum_{\alpha} E_{u}(\alpha)=\sum_{n} E_{u}(n), \\
E_{u}(\alpha) & =1 / 4 \mid \overline{\left.u_{\alpha}\right|^{2}}, \quad E_{u}(n)=\sum_{m=-n}^{n} E_{u}(\alpha),
\end{aligned}
$$

and similarly for $E_{v}$. The total kinetic energy spectrum is the sum of the two components. Clearly the distribution of kinetic energy with wavenumber so calculated is not the same as that of expression (11). In his paper, Baer argued that (for hemispheric data) the spectral amplitudes were approximately independent of $m$ at higher values of $n$ and that there was approximate equipartition of energy between the $u$ and $v$ components at these wavenumbers. The condition of no correlation between components $\langle\overline{u v}\rangle$ $=0$ was satisfied automatically because of the method of representing the hemispheric data.

Tang and Orszag (1978) argue that the velocity components should be expanded as

$$
(u, v)=\frac{1}{\cos \varphi} \sum_{\alpha}\left(\hat{u}_{\alpha}, \hat{v}_{\alpha}\right) Y_{\alpha}
$$

For the meridional velocity this results in a simple connection between $\hat{v}_{\alpha}$ and the streamfunction, namely

$$
\hat{v}_{\alpha}=i \frac{m_{\alpha} \psi_{\alpha}}{a}
$$

Tang and Orszag then assign the energy in wavenumber $\alpha$ in the meridional direction to the single wave with streamfunction $\psi_{\alpha}$. The energy expression

$$
E_{\varphi}=\left\langle\overline{1 / 2 v^{2}}\right\rangle=\frac{1}{4 \pi} \int_{-\pi / 2}^{\pi / 2} \int_{0}^{2 \pi} 1 / 2 \overline{v^{2}} \cos \varphi d \lambda d \varphi
$$

evaluated for the single wave gives, in terms of twosided spectral densities,

$$
E_{\varphi}(\alpha)=\frac{1}{4} \frac{(n+1 / 2)|m|}{a^{2}} \overline{\left|\psi_{n}^{m}\right|^{2}}
$$

The total energy at that wave number is

$$
E(\alpha)=\frac{1}{4} \frac{n(n+1)}{a^{2}} \overline{\left|\psi_{n}^{m}\right|^{2}}
$$

and the difference is assigned to the zonal component of the energy

$$
\begin{aligned}
E_{\lambda}(\alpha) & =E(\alpha)-E_{\varphi}(\alpha) \\
& =1 / 4 a^{-2}[n(n+1)-(n+1 / 2)|m|] \overline{\left|\psi_{n}^{m}\right|^{2}} .
\end{aligned}
$$

For isotropic turbulence, where $\psi_{n}^{m}$ is independent of $m$, an equal partition of energy between the two components follows. Thus, summing $E_{\varphi}(\alpha)$ and $E_{\lambda}(\alpha)$ over $m$ under this assumption gives

$$
\begin{aligned}
E_{\varphi}(n)=\sum_{m=-n}^{n} \frac{1}{4} \frac{(n+1 / 2)|m|}{a^{2}} \frac{\left.\psi_{n}^{m}\right|^{2}}{} & =1 / 8 a^{-2}[n(n+1)(2 n+1)] \overline{\left|\psi_{n}^{(m)}\right|^{2}}
\end{aligned}
$$

and

$$
\begin{aligned}
E_{\lambda}(n) & =E(n)-E_{\varphi}(n) \\
& =1 / 8 a^{-2}[n(n+1)(2 n+1) m] \overline{\left|\psi_{n}^{(m)}\right|^{2}}=E_{\varphi}(n) .
\end{aligned}
$$

The results above differ somewhat from those of Tang and Orszag whose formulas imply only an approximate equipartition at large $n$. This manner of assigning the energy to the velocity components, it must be noted, is not without arbitrariness. The "single wave" calculation, however, can be repeated directly with a complete spectrum since, under the assumption of homogeneity and isotropy, cross-product terms are uncorrelated (B, Section $3 b)$. These various expressions are evaluated from the data in a following section.

\section{c. Energy and enstrophy equations}

The equations of motion for the atmosphere may be written as

$$
\begin{array}{r}
\frac{\partial \mathbf{V}}{\partial t}+(\mathbf{V} \cdot \nabla) \mathbf{V}+f \mathbf{k} \times \mathbf{V}=D \\
C_{p} \frac{\partial T}{\partial t}+\mathbf{V} \cdot \nabla C_{p} T=Q,
\end{array}
$$

where terms on the left-hand-side of the equations apply to the non-divergent winds only and all divergent effects together with source and sink terms are lumped together on the right-hand sides.

The transformed kinetic energy equation is written as

$$
\frac{\partial E_{n}}{\partial t}=I_{n}+2\left[\alpha_{n}-\nu n(n+1) a^{-2}\right] E_{n}
$$

in parallel with (2) where $\alpha_{n}$ is taken to be a source/ sirk term including all divergent effects and sources and sinks other than the explicit molecular viscous dissipation term.

It follows that the enstrophy equation may be written by analogy with (3) as 


$$
\frac{\partial G_{n}}{\partial t}=J_{n}+2\left[\alpha_{n}-\nu n(n+1) a^{-2}\right] G_{n},
$$

where the interaction terms are obtained from the non-divergent part of the flow as

$$
\begin{aligned}
J_{n} & =-1 / 4 \sum_{m=-n}^{n}\left[\zeta_{\alpha}^{*}\{\mathbf{V} \cdot \nabla \zeta\}_{\alpha}+\zeta_{\alpha}\{\mathbf{V} \cdot \nabla \zeta\}_{\alpha}^{*}\right] \\
& =1 / 4 \frac{n(n+1)}{a^{2}} \sum_{m=-n}^{n}\left[\psi_{\alpha}^{*}\{J(\psi, \zeta)\}_{\alpha}+\psi_{\alpha}\{J(\psi, \zeta)\}_{\alpha}^{*}\right]
\end{aligned}
$$

and

$$
I=\frac{a^{2}}{n(n+1)} J_{n} .
$$

[These relationships are used in Chen and Wiin-Nielsen (1978) although the printed versions are incorrect.]

The transformed available potential energy equation is written symbolically as

$$
\frac{\partial A_{n}}{\partial t}=M_{n}+2 \beta_{n} A_{n},
$$

where $M_{n}$ is the non-divergent interaction term,

$$
M_{n}=-1 / 4 C_{p} \gamma \sum_{m=-n}^{n}\left[T_{\alpha}^{*}\{\mathbf{V} \cdot \nabla T\}_{\alpha}+T_{\alpha}\{\mathbf{V} \cdot \nabla T\}_{\alpha}^{*}\right]
$$

and $\beta_{n}$ is taken to represent divergent effects and source-sink terms.

Eqs. (14)-(16) parallel those of B (Section 5) although they are obtained in a rather different fashion. In particular, these equations are obtained from the atmospheric equations where non-barotropic terms are lumped together with source and sink terms and where the spectra and interaction terms are obtained from data by spatial and time averaging.

In an inertial subrange

$$
0=\frac{\partial E_{n}}{\partial t}=I_{n}=2\left[\alpha_{n}-\nu \frac{n(n+1)}{a^{2}}\right] E_{n},
$$

with similar relations for enstrophy. For atmospheric scales where viscosity is small, $\alpha_{n}$ is taken to represent divergent effects including conversions from available potential energy.

As previously, the inertial transfer terms, $I_{n}, J_{n}$, $M_{n}$, serve to redistribute energy and enstrophy between wavenumbers, not to change the total amount. Flux functions are defined in analogy with the plane turbulence case. In this case, however, the scale parameter is no longer continuous and a difference formula is adopted.

The fluxes are defined by the equations

$$
\left.\begin{array}{rl}
I_{n} & =-a\left(F_{n}-F_{n-1}\right) \\
J_{n} & =-a\left(H_{n}-H_{n-1}\right) \\
M_{n} & =-a\left(P_{n}-P_{n-1}\right)
\end{array}\right\},
$$

and are calculated from the interaction terms using

$$
F_{n+1}=-a^{-1} \sum_{l=1}^{n} I_{l},
$$

and similarly for $H$ and $P$. The inertial constraint that the effect of the interactive terms is to redistribute energy only requires that

$$
\sum_{n=0}^{\infty} I_{n}=\sum_{n} J_{n}=\sum_{n} M_{n}=0
$$

\section{d. Parameterization of unresolved scales}

The discussion of Section $3 \mathrm{c}$ passes over directly to the case of spherical harmonic representation. The total inertial term is thought of as being composed of a resolved and unresolved part,

$$
I_{n}=I_{n}^{R}+I_{n}^{U},
$$

where $I_{n}^{R}$ is calculated using (16) where the streamfunction representation is truncated at some $n=N$. The condition

$$
\sum_{n=0}^{\infty} I_{n}=0=\sum_{n=0}^{N} I_{n}^{R}+\sum_{n=0}^{\infty} I_{n}^{U}
$$

requires that $\sum_{n=0}^{\infty} I_{n}^{U}=0$ since in the non-divergent case $\sum_{n=0}^{N} I_{n}^{R}=0$ algebraically (Platzman, 1960).

In an inertial subrange the resolved and unresolved interaction terms must add to zero, i.e.,

$$
I_{n}^{R}=-I_{n}^{U},
$$

Following Leith (1971) an estimate of the unresolved transfer for an enstrophy cascading subrange is used in the form

$$
L_{n}=I_{n}^{U}=-2 \eta^{1 / 3} f_{n} E_{n} .
$$

Similar expressions are used for enstrophy and available potential energy.

\section{Analysis of observational data}

\section{a. Data sources}

The data used in this study consist of the wind and temperature fields for January and July 1979 from the FGGE-IIIa global data set. These data are global operational analyses produced by the National Meteorological Centre (McPherson et al., 1979). The data are available on 12 pressure levels $(1000,850$, 
$700,500,400,300,250,200,150,100,70,50 \mathrm{mb})$ and at the two standard synoptic times.

The streamfunction is obtained from the winds at grid points by numerical quadrature. In terms of the scaled velocity components

$$
(U, V)=(u, v) \frac{\cos \varphi}{a},
$$

the vorticity is

$$
\zeta=\frac{1}{\cos ^{2} \varphi}\left(\frac{d V}{\partial \lambda}-\cos ^{2} \varphi \frac{\partial U}{\partial \varphi}\right) .
$$

The spectral coefficients of $\zeta$ are obtained by transforming this expression in the manner of Bourke (1972). The method combines the calculation of the derivatives and the transformation in a manner that is exact for grid data at Gaussian latitudes which are consistent with a suitably truncated spherical harmonic series. In this application the data are interpolated to the appropriate Gaussian latitudes. The results pressented all apply to January unless explicitly noted otherwise. Results for July are qualitatively similar to those for January except as noted and displayed for comparison.

\section{b. Spectra and spectral slopes}

The spectra of kinetic energy, enstrophy and available potential energy are obtained in terms of the two-dimensional scale parameter $n$ using (10)-(13). These spectra are obtained from data of global extent whereas those of Baer $(1972,1974)$ and Chen and Wiin-Nielsen (1978) were obtained from Northern Hemisphere data only. Lambert (1981) also used global data.

The spectra are further decomposed into stationary and transient parts under time averaging. In the case of enstrophy, where the overbar represents the time average (in this case a month) and the prime the deviation therefrom,

$$
\begin{aligned}
G(n) & =1 / 4 \sum_{m=-n}^{n} \overline{\left|\zeta_{\alpha}\right|^{2}} \\
& =1 / 4 \sum_{m=-n}^{n} \overline{\left.\zeta_{\alpha}\right|^{2}}+1 / 4 \sum_{m=-n}^{n} \overline{\left|\zeta_{\alpha}^{\prime}\right|^{2}} \\
& =G_{S}(n)+G_{T}(n) .
\end{aligned}
$$

The vertically integrated spectra together with the stationary and transient components for January are displayed in Fig. 1. Spectra for both January and July are compared in Fig. 2.

The vertically integrated spectra for January qualitatively resemble the hemispheric results of Chen and Wiin-Nielsen. The most notable differences occur in the low wavenumber regions of the spectra. For the shorter wavelength region the qualitative correspondence extends to the behavior of the spectra at about $n=25$ where a drop in spectral amplitudes is seen. This is particularly noticeable in the enstrophy spectrum and is perhaps even more pronounced in the present January case than was the case in the study of Chen and Wiin-Nielsen. They attributed this behavior to smoothing of the initial data, and this is certainly a plausible suggestion.

The decomposition of the spectra into stationary and transient parts illustrates rather dramatically that two rather different regimes exist in wavenumber space: The high-wavenumber regime, $n>8-10$, is characterized by a spectral power-law of the form $E$ $\propto n^{-b}$ and is dominated by the transient component. The low-wavenumber regime lacks power-law behavior and is dominated by the stationary component. This stationary part of the flow at low wavenumbers is in turn dominated largely by components with zonal symmetry (i.e., the $m=0$ components).

Fig. 2 shows that the spectrum of the high-wavenumber, power-law transient regime is remarkably insensitive to the time of year, while the low-wavenumber stationary regime undergoes marked changes between January and July. These results reinforce the ideas, implicit in most discussions of large-scale atmospheric flow, that the stationary component, assumed to be largely forced by topography and heat sources, dominates the large-scale flow while smaller scales are dominated by the transient component which might be expected to behave in accordance with the turbulence theory.

The insensitivity of the high-wavenumber region of the spectrum to the time of year together with the power-law behavior of the spectrum and the dominance of the transient component suggests that this wavenumber region might be characterized as a freely interacting field of turbulent eddies and hence might be expected to obey, at least to some extent, the constraints of simple turbulence theory.

If this range corresponds to the enstrophy cascading inertial subrange of two-dimensional or geostrophic turbulence, -3 power laws are expected for kinetic and available potential energy while enstrophy would exhibit a -1 power law. For the purposes of comparison, the slopes of the kinetic energy and available potential energy spectra for the possible inertial range $14 \leqslant n \leqslant 25$ are shown in Fig. 3 together with those from the studies of Baer $(1972,1974)$ and Chen and Wiin-Nielsen (1978). These previous studies were based on hemispheric data and Baer's kinetic energy results were obtained by analysis of the wind components themselves rather than the streamfunction.

The most obvious feature of the current analysis is the lesser slopes obtained for this spectral region at virtually all levels of the atmosphere. For kinetic 


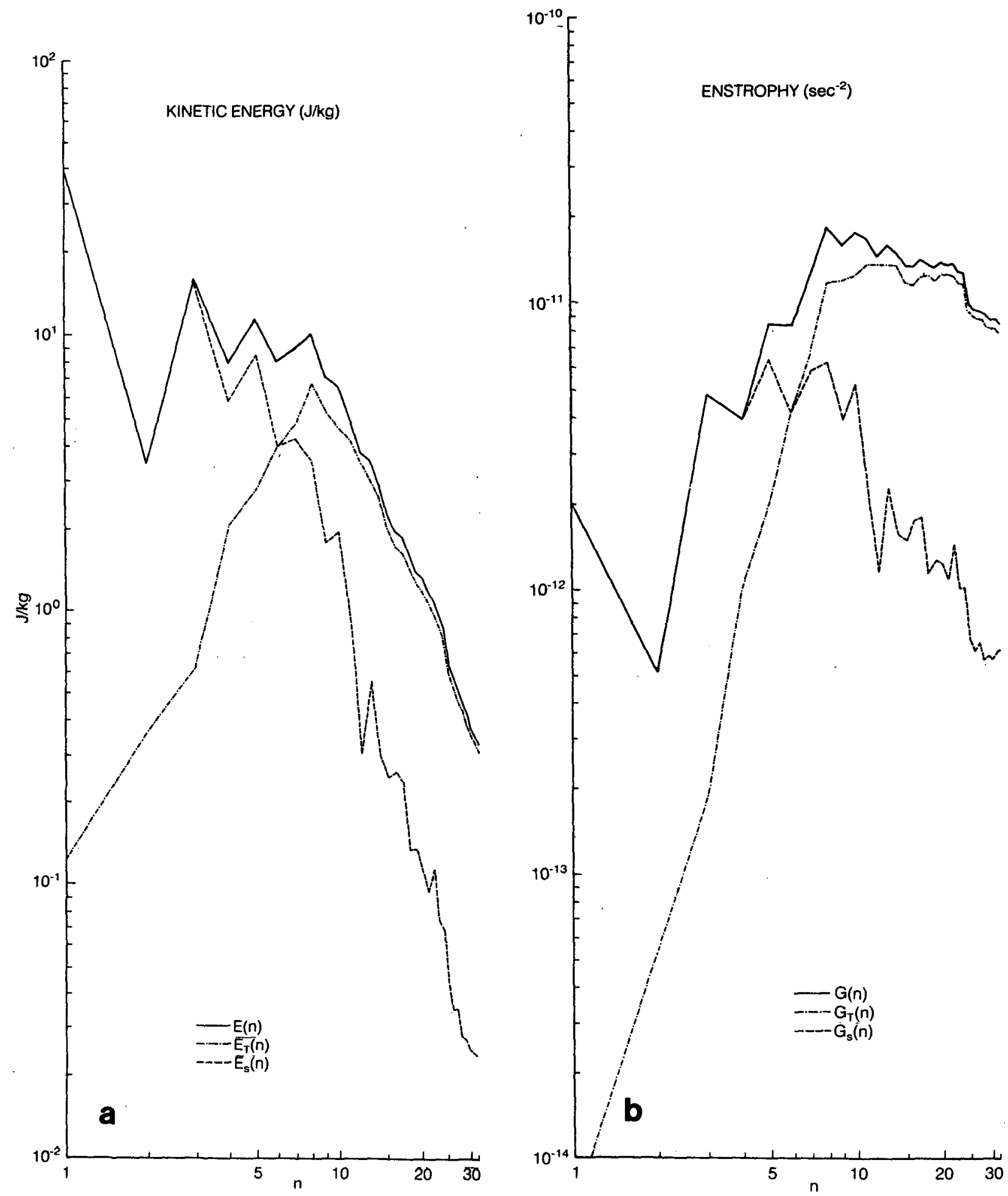

FIG. 1. Integrated spectra of (a) kinetic energy $E(n)$, (b) enstrophy $G(n)$, and (c) available potential energy $A(n)$, for January. Stationary and transient components are also shown.

energy the slopes obtained in this study show an increase with height to a maximum near $200-250 \mathrm{mb}$ and a subsequent decrease. This resembles the results reported by Baer (1972), but the magnitudes of the slopes are considerably smaller. Chen and Wiin-Nielsen, on the other hand, have a region of nearly con- 


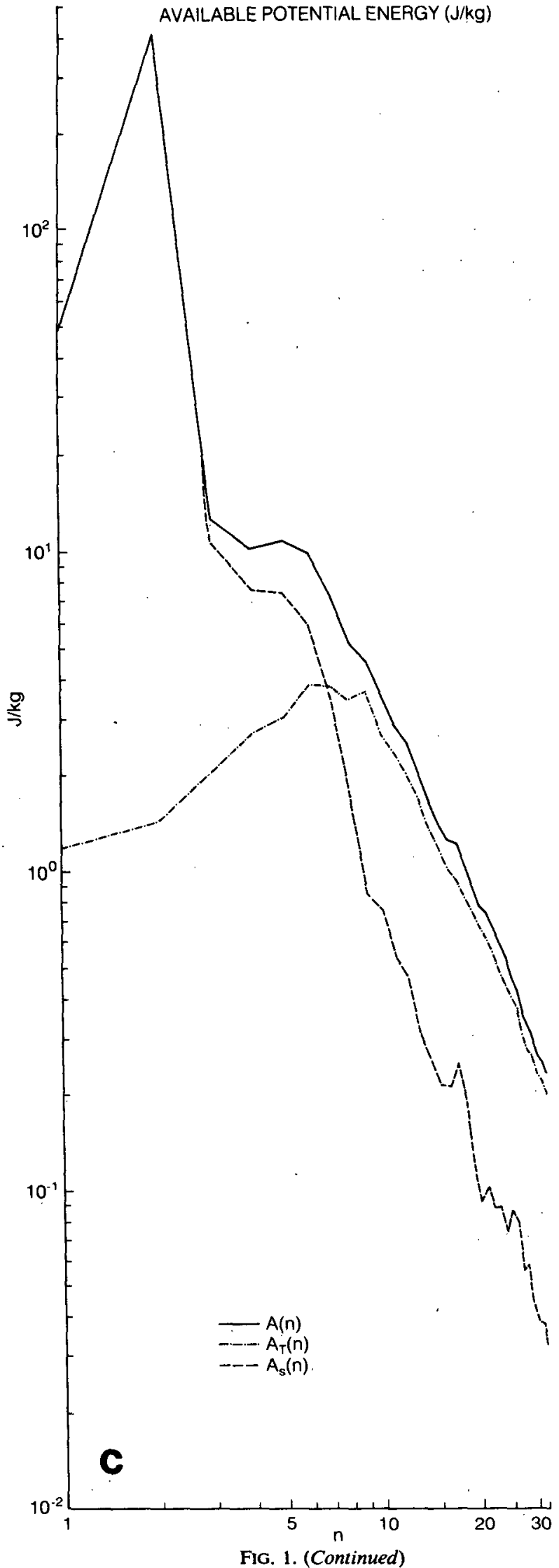

stant slope throughout the range $500-200 \mathrm{mb}$. For available potential energy, the shape of the curves are similar below $200 \mathrm{mb}$ in the three cases although the slopes obtained in this study are again smaller than those obtained previously. Above $200 \mathrm{mb}$, however, the spectral slopes obtained in this study decrease, while they increase in the results of previous studies.

While the "slope" of the spectrum over a restricted wavenumber range is a rather sensitive parameter, it is nevertheless clear that the results of this study differ from those of previous studies. Further calculation shows that these differences in spectral slope are not attributable to the difference between global and hemispheric analyses. They must therefore be ascribed to differences in the data used in the various studies.

For the purposes of comparison with theory, the spectral slopes obtained from the data are somewhat shallower than the values of -3 suggested by simple theory. It must be emphasized, however, the enstrophy-cascading inertial subrange is not really a prediction for the atmosphere but is a possible solution to the spectral equation in an unforced subrange which may or may not have some correspondence to the situation in the real atmosphere. Consequently, the fact that the spectra obey power laws at all may be considered to be a striking, although by now well known, feature of the atmosphere.

In the low-wavenumber regime, on the other hand, there seems to be nothing that suggests a correspondence to the $-5 / 3$ power law, energy-cascading inertial subrange. The transient component is comparatively weak in this regime, perhaps surprisingly so. The stationary component dominates the flow and the identification with large-scale forced modes is supported by the difference in the spectra between January and July corresponding, it is presumed, to differences in forcing between the hemispheres. As might be expected, the variation in the spectrum of available potential energy is less marked than that of kinetic energy.

The pressure-wavenumber cross section of kinetic energy is displayed in Fig. 4. The contours are drawn at roughly logarithmic intervals. A maximum of energy is found at the $200-300 \mathrm{mb}$ level. This is in fact the level for which the spectral slopes in the kinetic energy are closest to -3 . The slope of the spectrum in the high-wavenumber region is apparently steeper at levels where the kinetic energy is larger.

The kinetic energy displays a notable maximum at all levels at wavenumber 3 . A secondary maximum at wavenumber 8 is also of note and occurs at the wavenumber of the maximum of the transient component, although the contribution to the total kinetic energy from the stationary component is still appreciable. One of the more interesting and important aspects of the study of large-scale atmospheric turbulence must be the nature of the interaction between these two flow regimes. 


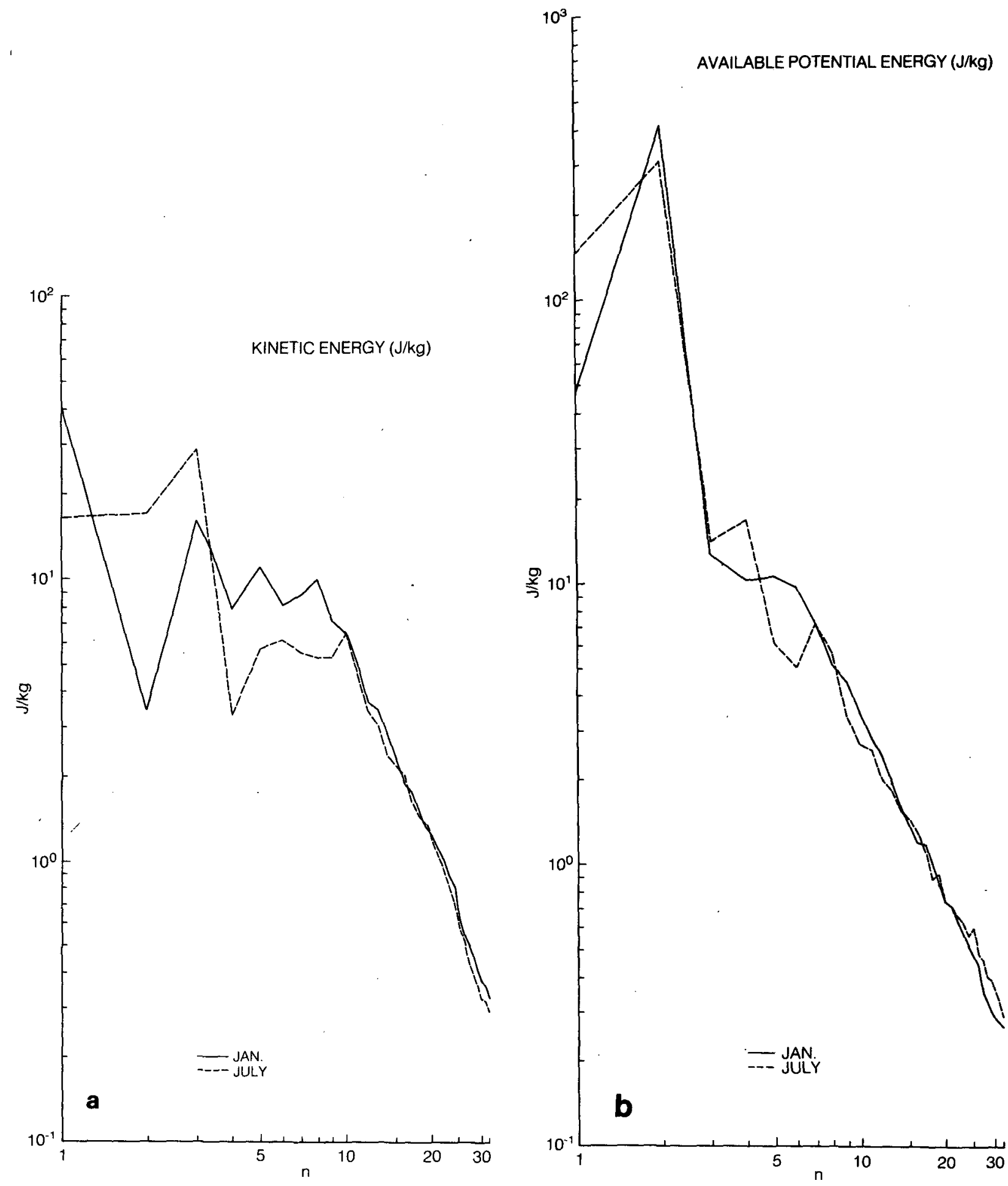

FIG. 2. Integrated kinetic energy spectra (a) and available potential energy spectra (b) for January and July.

\section{c. Homogeneity and isotropy}

Two-dimensional turbulence theory is usually formulated under the assumption of homogeneity and isotropy. If the identification of the high-wavenum- ber, transient-dominated region of the spectrum with simple turbulent behavior is at all valid, this region of the spectrum should also display the characteristics of homogeneity and isotropy.

As pointed out in B, a necessary condition is that 

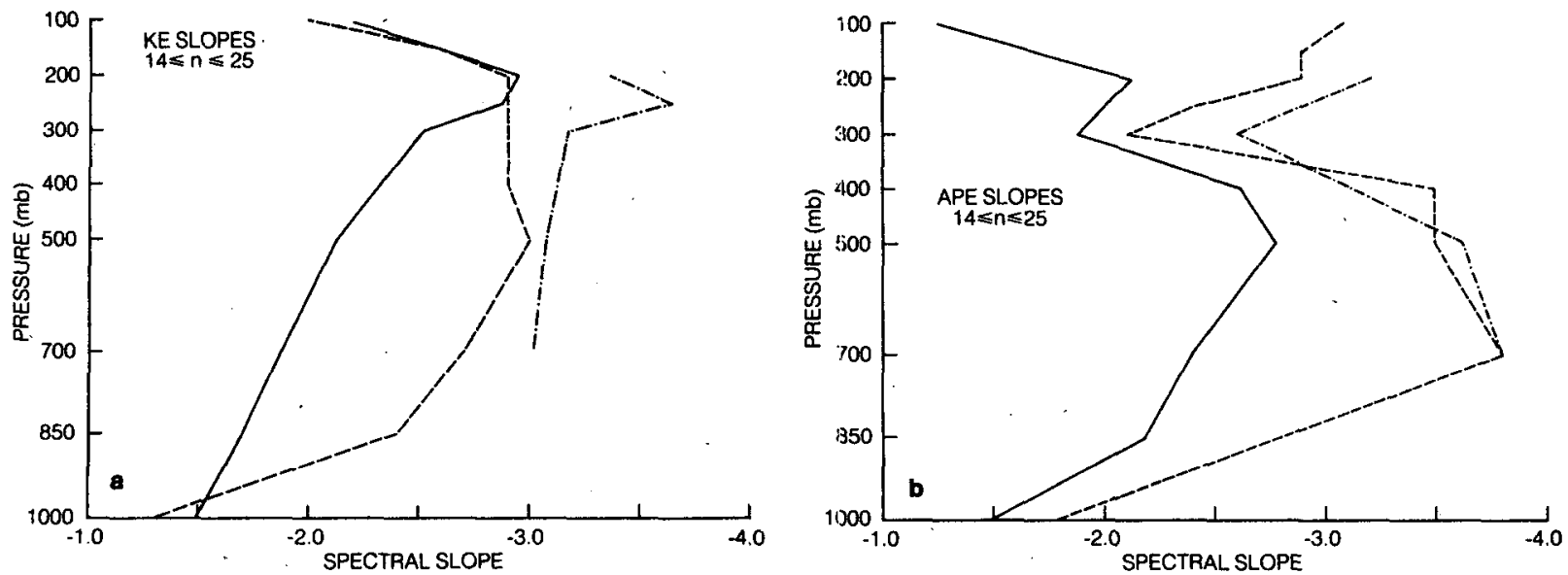

FIG. 3. Slope of straight line fitted to the kinetic energy spectrum (a) and available potential energy spectrum (b) in the wavenumber region $14 \leqslant n \leqslant 25$ of Fig. 1. Value of $-b$ is in the power law $E=A n^{-b}$. Dash-dotted line presents the results of Baer (1972), dashed line the results of Chen and Wiin-Nielsen (1978), and solid line results of this study using global data.

$\overline{\left.\psi_{n}^{m}\right|^{2}}$ is independent of $m$. Vertically integrated and time-averaged values of the one-sided energy spectral densities

$$
E_{n}^{m}=\frac{1}{2} \frac{n(n+1)}{a^{2}} \overline{\left.\psi_{n}^{m}\right|^{2}}
$$

are shown in Fig. 5. For $n \geqslant 10$, the isopleths are reasonably horizontal over most of the range of $m$, although energy densities tend to drop off near the edge of the triangle. A plot of available potential energy is also shown. There is some evidence for isotropy in this quantity, also, at least in the highwavenumber regime, although it is not as convincing as for kinetic energy. Baer (1972, 1974) displayed sim-

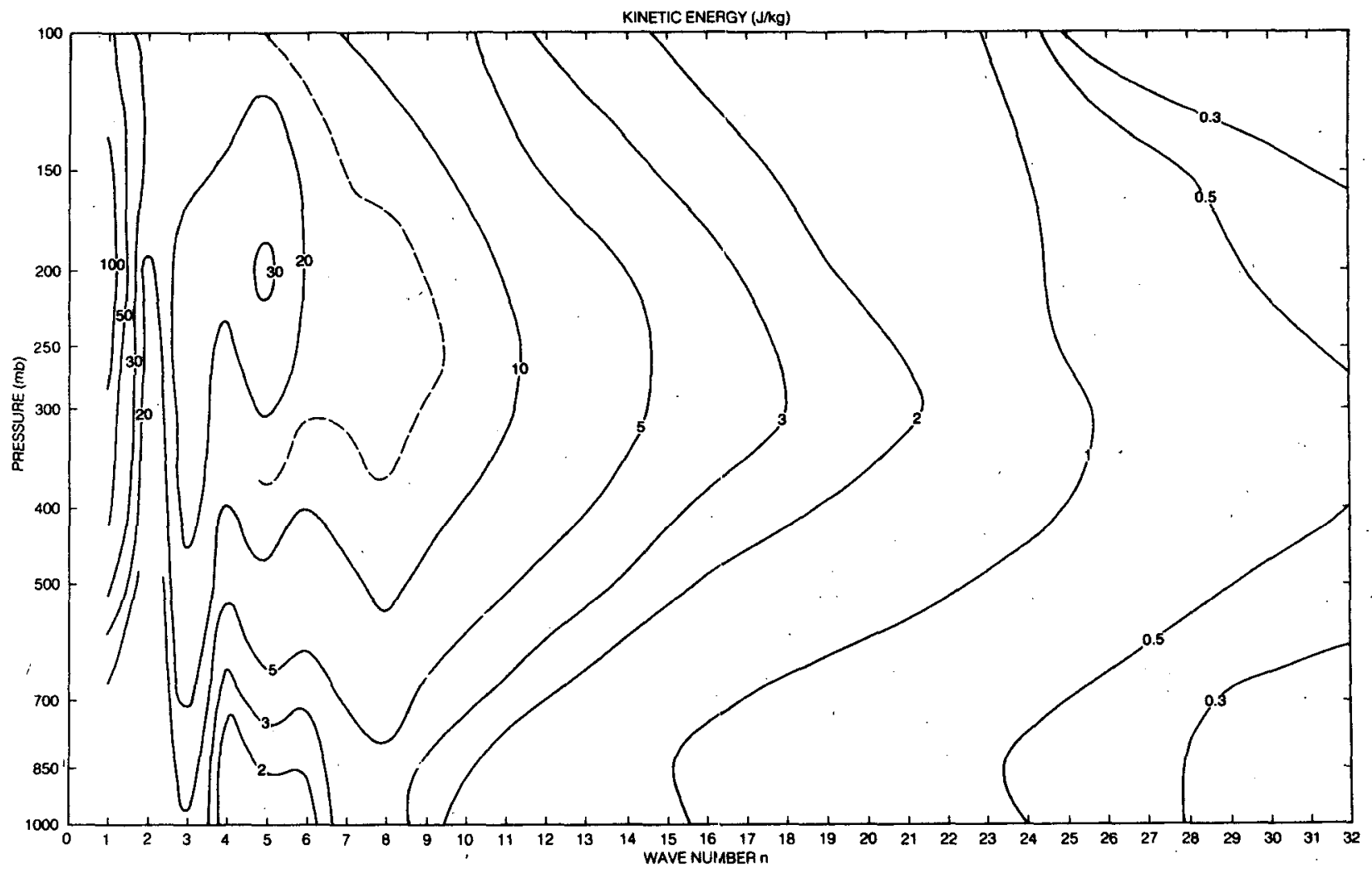

FiG. 4. The kinetic energy spectrum for January as a function of pressure. 


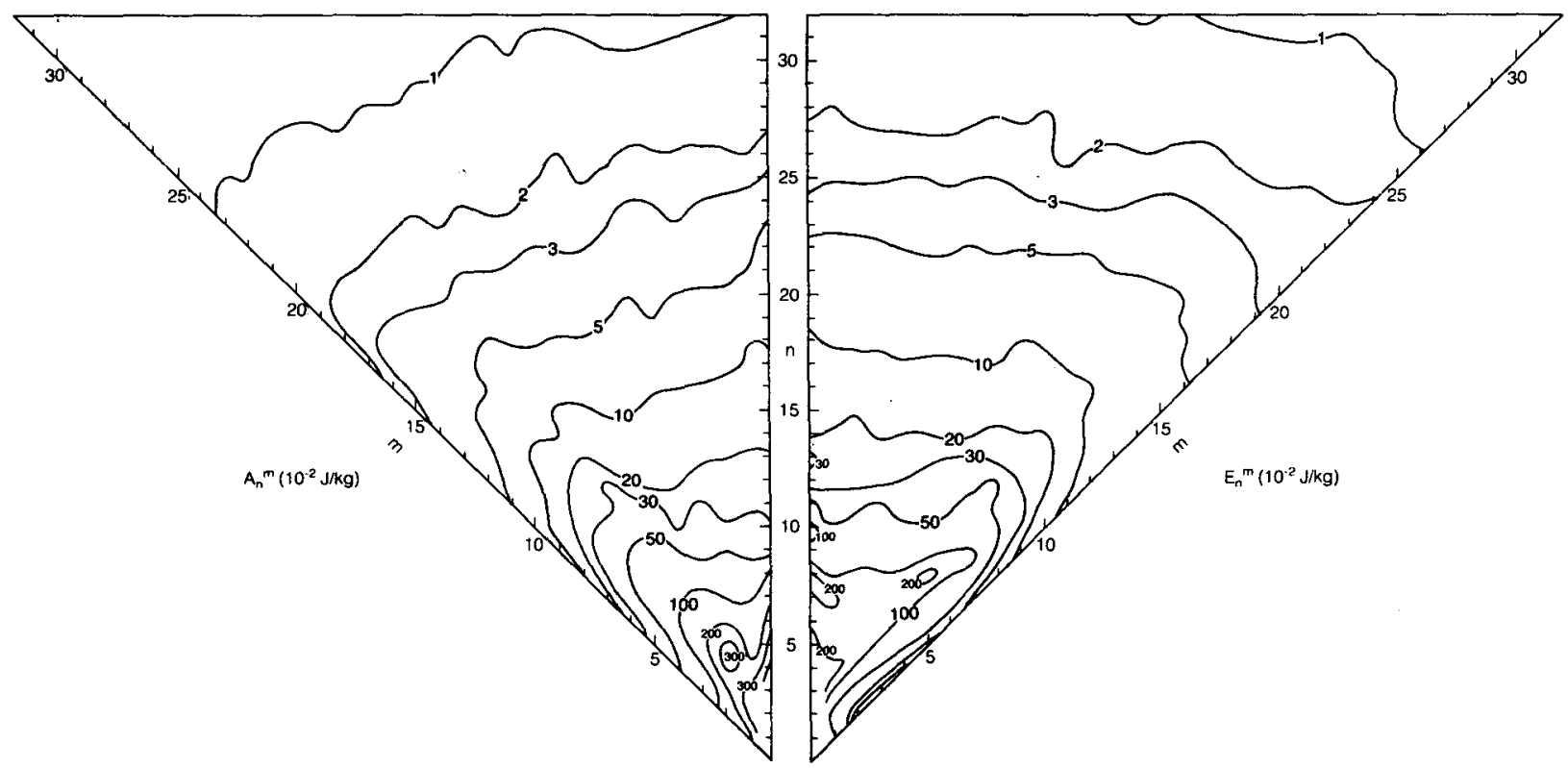

FIG. 5. Integrated kinetic and available potential energy spectra for January.

ilar diagrams obtained from hemispheric data where the kinetic energy was expressed in terms of the velocity components.

The partitioning of the kinetic energy between velocity components may be accomplished at least in two ways as discussed in Section 4b. Perhaps neither of these approaches is truly satisfactory. Fig. 6 displays the velocity components spectra $E_{u}(n), E_{v}(n)$, in the manner of Baer (1972) together with the cospectrum defined as

$$
\langle\overline{u v}\rangle=\sum_{\alpha} 1 / 2 \overline{u_{\alpha} v_{\alpha}^{*}}=\sum_{n} E_{u v}(n) .
$$

Fig. 7 displays the spectra of the velocity components in the manner of Tang and Orszag. These spectra together with that of available potential energy are plotted for $n \geqslant 10$ in Fig. 8.

These figures demonstrate a number of features of the flow and of the manner of calculating the spectra. Both Figs. 6 and 7 illustrate that in the high-wavenumber regime there is approximate equipartition of energy between components defined in either fashion. The cospectrum term is small as well. Not only are the kinetic energy components in approximate equipartition but so also is the available potential energy. The meridional components $E_{v}(n)$ and $E_{\varphi}(n)$ of kinetic energy calculated in either fashion are remarkably similar at all wavenumbers. That these necessary conditions hold supports the existence of a regime of approximately homogeneous and isotropic turbulence at higher wavenumbers.

In the low-wavenumber regime equipartition no longer holds even approximately. It is also clear that
$E_{u}(n) \neq E_{\lambda}(n)$ at these wavenumbers illustrating the different assignment of energy among wave numbers in the two approaches.

\section{d. Nonlinear interactions and fluxes of energy and enstrophy}

The nonlinear interaction terms $I_{n}, J_{n}$ and $M_{n}$ represent the interchange of energy and enstrophy between wavenumbers. They may be thought of as being composed of two parts, as discussed in Section $4 d$. One part is calculable from the data and represents the "resolved" part of the interaction between wavenumbers while the second part is unavailable from the data and represents the effects on the resolved scales of scales of motion not contained in the data.

The resolved part of the vertically integrated, nonlinear interaction terms for January are displayed in Fig. 9. Values for July are quantitatively similar. The associated flux functions for January and July together with the values for January using data from the Northern Hemisphere only are plotted in Fig. 10. The distribution with pressure of the resolved kinetic energy flux function is shown in Fig. 11.

Values of the resolved interaction terms $I_{n}^{R}$ and $M_{n}^{R}$ for January and July are given in Table 1 . Differences are largely though not completely, confined to the low-wavenumber region. Using (18) the sum of these values are the fluxes of Fig. 10. In a stationary situation Eq. (14) implies that the net interaction term is equal and opposite in sign to the source-sink terms. If it is presumed that $I_{n}^{U}$ is relatively small, then $I_{n}^{R}$ of Fig. 9a is just the negative of the source-sink term. 


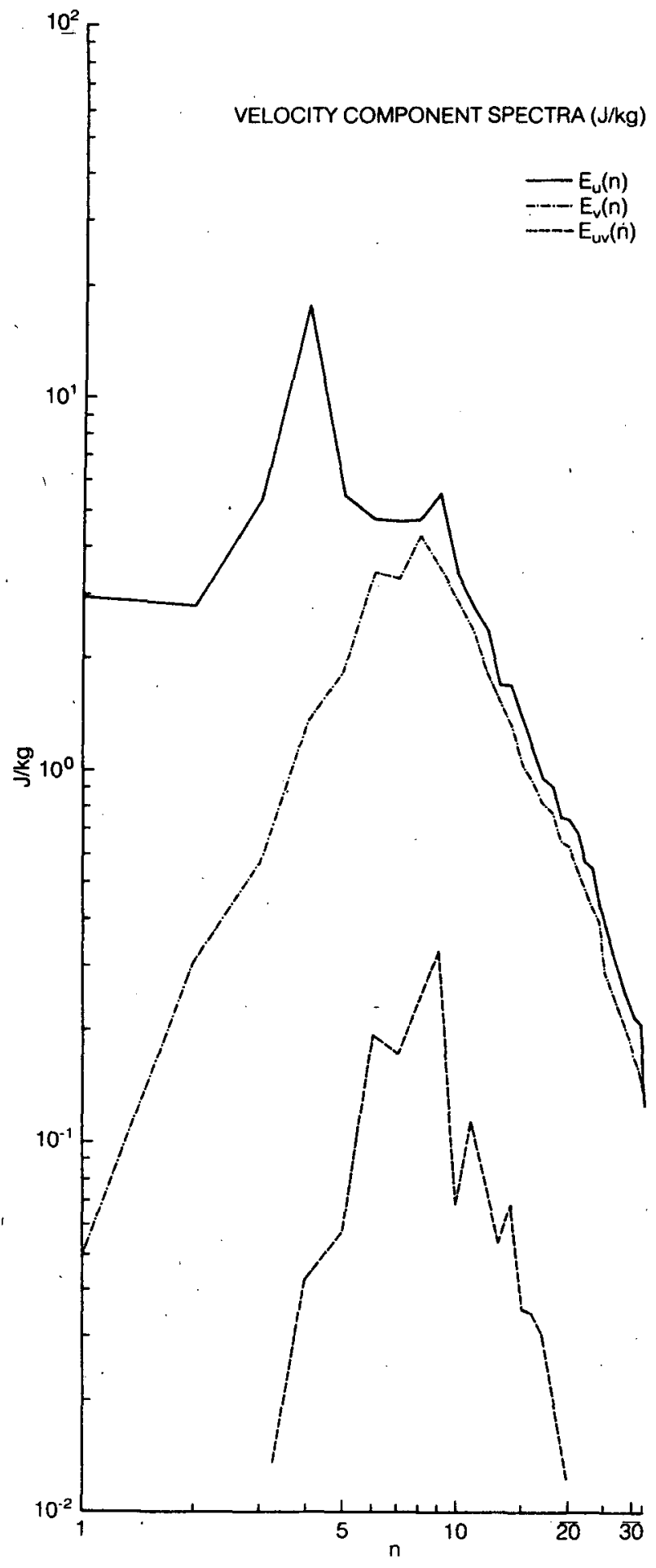

FIG. 6. Integrated spectra of the velocity components and the associated cospectrum for January.

In this calculation the source-sink term includes the effects of the divergent part of the flow. Similar remarks apply for $J_{n}^{R}$ and $M_{n}^{R}$.

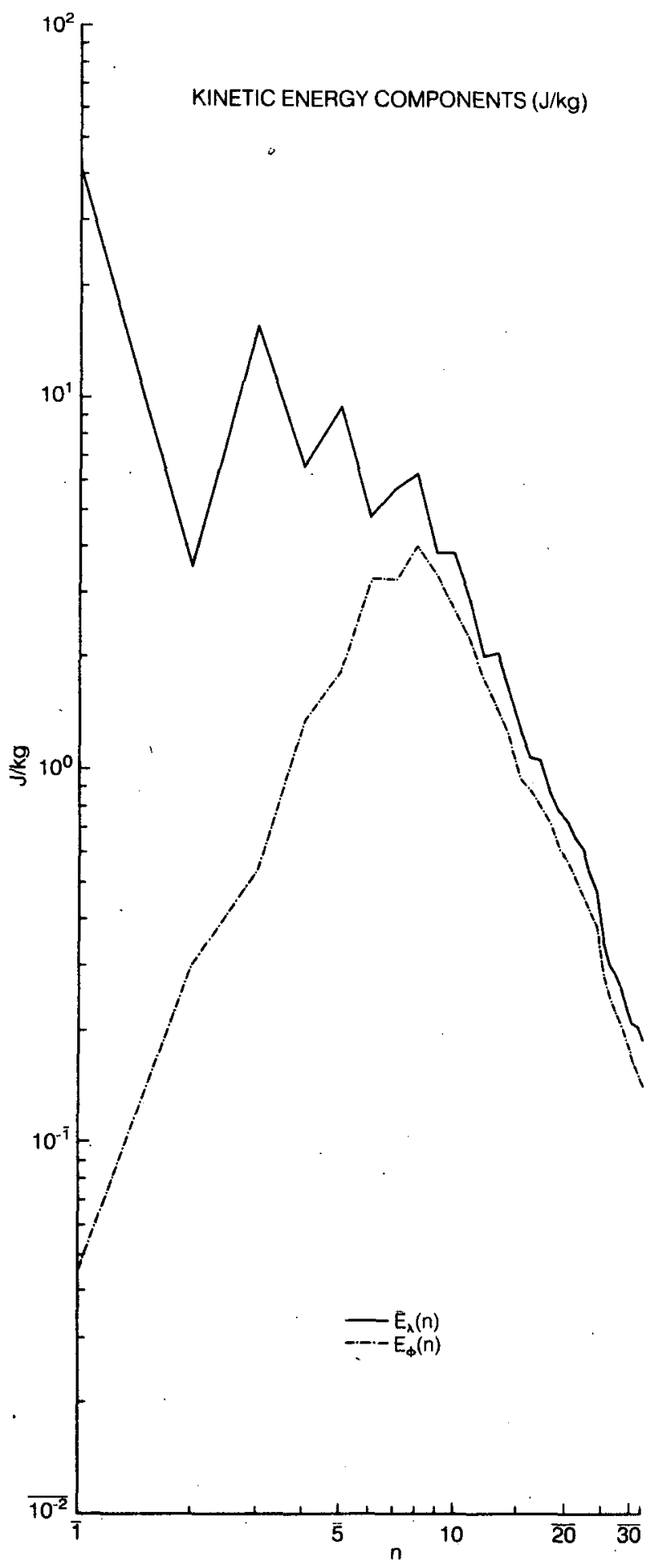

FIG. 7. Integrated zonal and meridional kinetic energy spectra for January.

Interpreted in this way, the figures show a broad source of energy and enstrophy at "intermediate" wavenumbers and a strong sink of kinetic energy at 


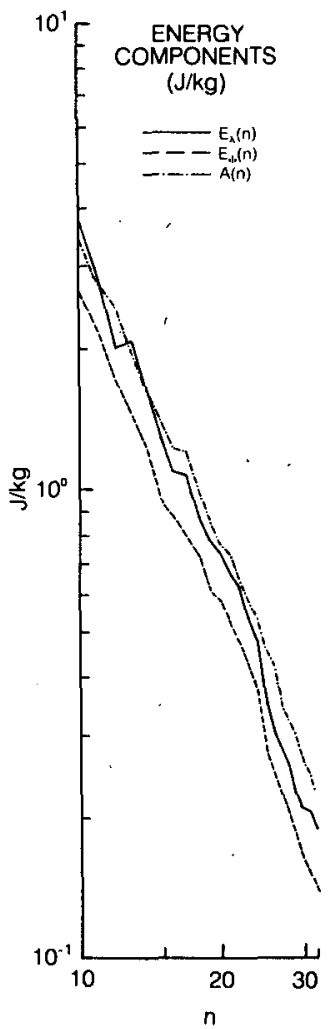

FiG. 8. Integrated kinetic energy component spectra together with the available potential energy spectrum for the high-wavenumber transient regime for January. low wavenumbers and of enstrophy at high wavenumbers, in general agreement with the usual ideas of atmospheric behavior. These source and sink regions are connected by the (resolved) fluxes through wavenumber space of energy and enstrophy, as shown in Fig. 10. The maximum fluxes occur in the upper troposphere (Fig. 11) at the level of maximum spectral amplitude (Fig. 4). This is also the level at which spectral slopes are steepest (Fig. 3). For available potential energy $M_{n}^{R}$ (Fig. 9c) is dominated by a large value at $n=2$. There is a large source of available potential energy at this wavenumber and a broadly distributed sink at higher wavenumbers. The associated down-scale flux of available potential energy is shown in Fig. 10c.

The results of the calculations for January are similar to those of Chen and Wiin-Nielsen in a general way but differ considerably in magnitude and detail. The calculations for the Northern Hemisphere especially should be comparable. Considerably smaller values of interaction terms and fluxes are calculated here, differing from those of Chen and Wiin-Nielsen by about a factor of 2 . As well, the maximum value of the interaction term $I_{n}^{R}$ (Fig. 9a) occurs at $n=2$ in the Chen and Wiin-Nielsen calculation but at $n$ $=3$ in the calculations performed here.

It is interesting to note that the energy flux to larger scales (Figs. 10a, 11) decreases sharply for $n \leqslant 5$. It is tempting to ascribe this feature of the observed flow to the wave domain cutoff feature of geostrophic tur-

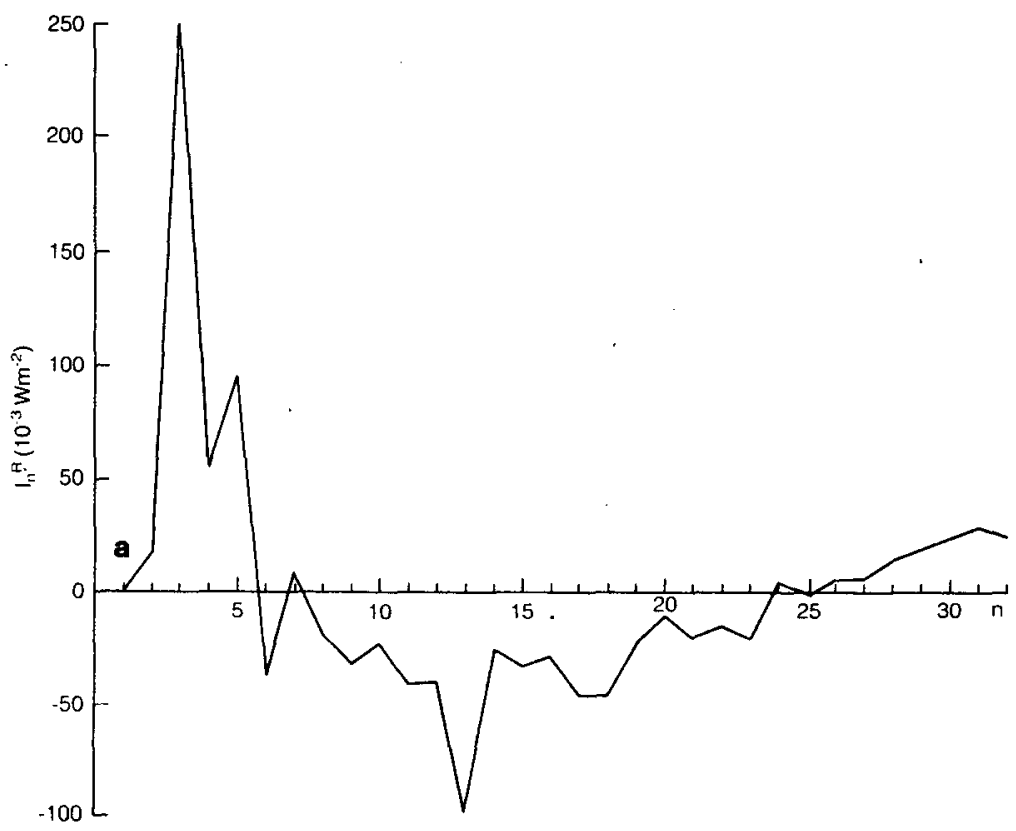

FIG. 9a. Integrated values of the resolved part of the kinetic energy nonlinear interaction term for January. Positive values indicate that energy is being transferred to that wavenumber from other scales. 


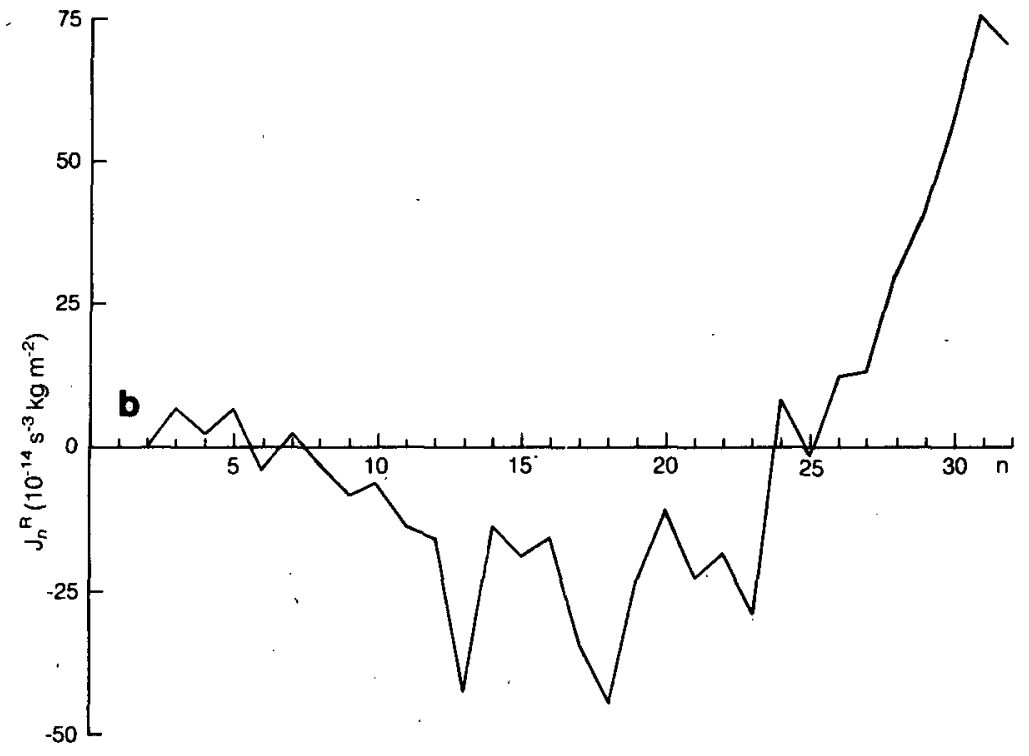

FIG. 9b. As in Fig. 9a but for enstrophy.

bulence described by Rhines (1975) and produced in numerical turbulence experiments by Rhines (1979) and Basdevant et al. (1981). Atmospheric conditions at these scales do not clearly parallel those of the theory, however, so the correspondence may be more apparent than real.

\section{e. Inertial subranges}

One of the reasons for calculating the various turbulence statistics presented in this study is in order to weigh the evidence for the existence of an inertial subrange, at least in the higher wavenumber regime. The existence of an enstrophy cascading inertial subrange has a variety of consequences for understanding and modelling atmospheric flow. In particular, the existence of such a subrange would provide some information and justification for choosing a particular density of the atmospheric observational network and for choosing a particular truncation when modelling atmospheric flow. The effects of unresolved scales in observations and models could supposedly be properly parameterized if the existence of an inertial subrange could be demonstrated.

The requirements of a pure two-dimensional enstrophy-cascading subrange include isotropy, equipartition of energy, a -3 slope for kinetic energy, zero flux of kinetic energy and constant flux of enstrophy. It has been argued that at higher wavenumbers, isotropy and equipartition do appear to be features of the atmosphere, and while the kinetic energy slope is somewhat less than -3 a clear power law regime

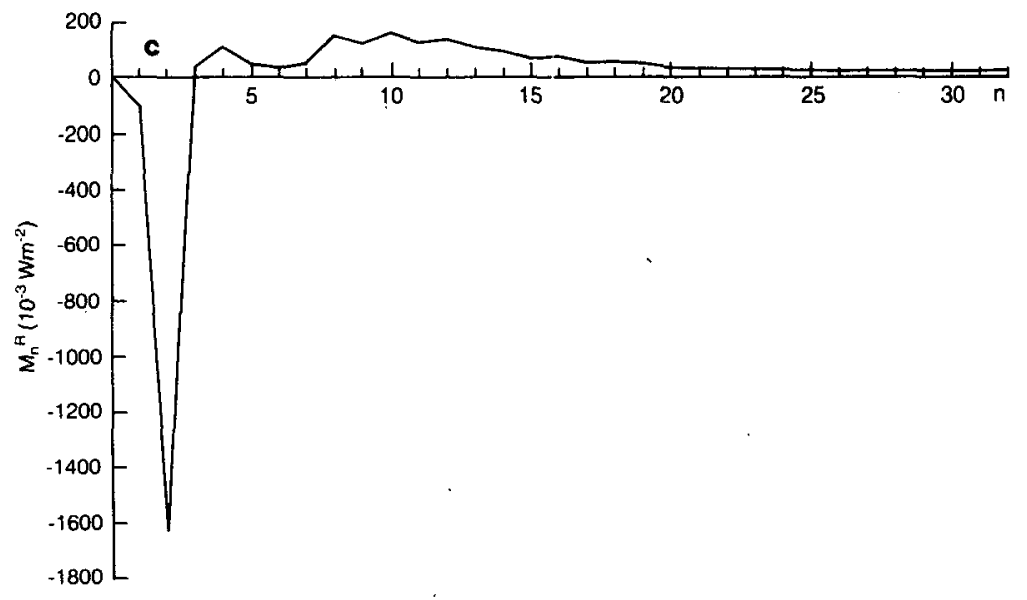

FIG. 9c. As in Fig. 9a but for available potential energy. 


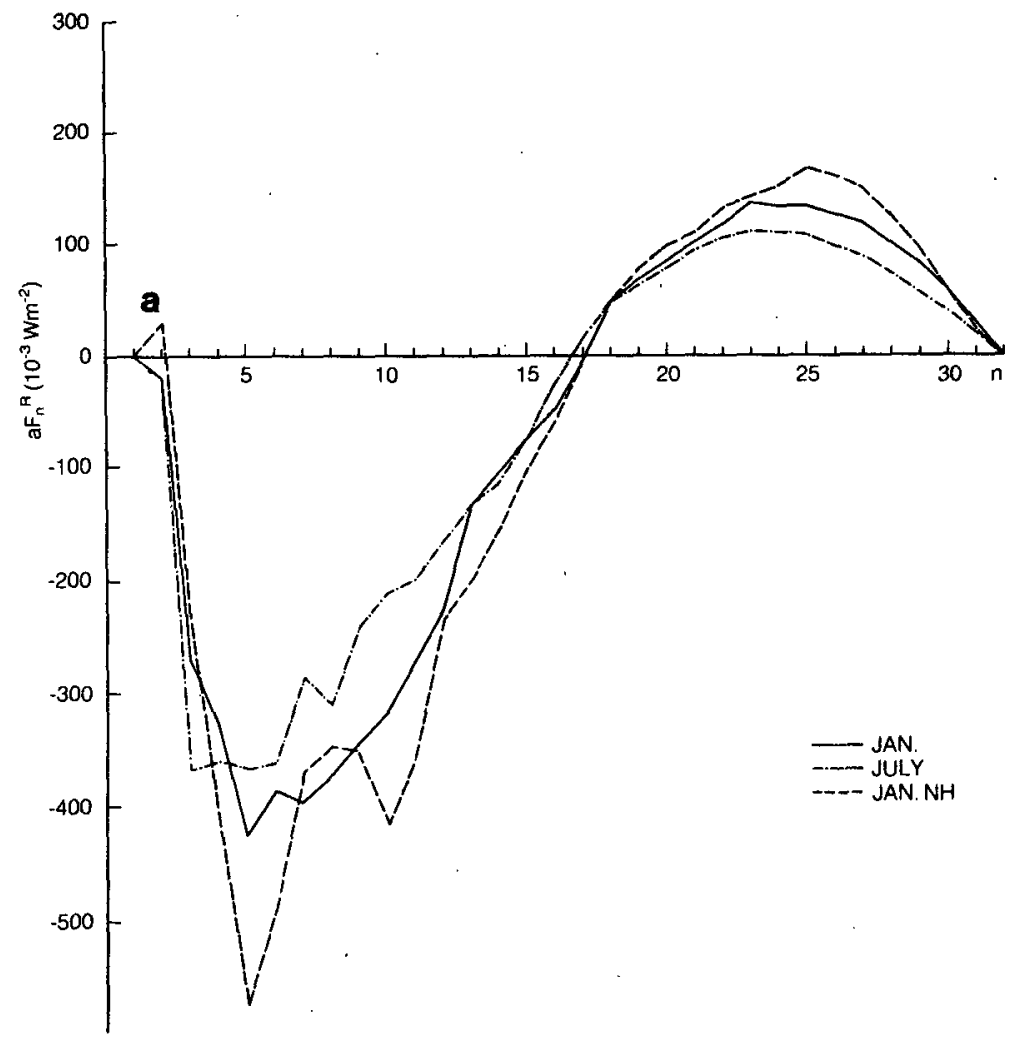

FiG. 10a. Integrated values of the resolved part of the nonlinear kinetic energy flux term. Negative values imply upscale transfer from higher to lower wave numbers.

of turbulent flow exists. The calculated fluxes of en- that these resolved fluxes are not inconsistent with ergy and enstrophy include only the resolved part of the behavior expected in an inertial subrange and that the total flux so they cannot exhibit the behavior expected in a subrange. Nevertheless, it can be argued $F_{n}^{R}$ is small enough that the effect of unresolved scales could cancel it while $H_{n}^{R}$ is large enough so that the

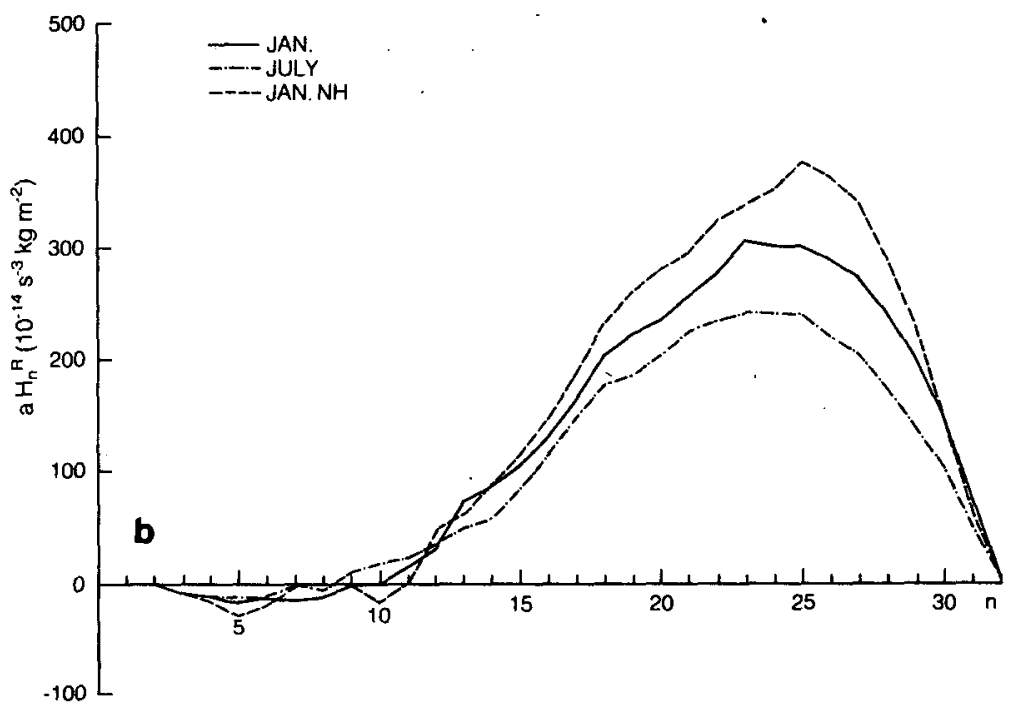

FIG. 10b. As in Fig. 10a but for enstrophy. 


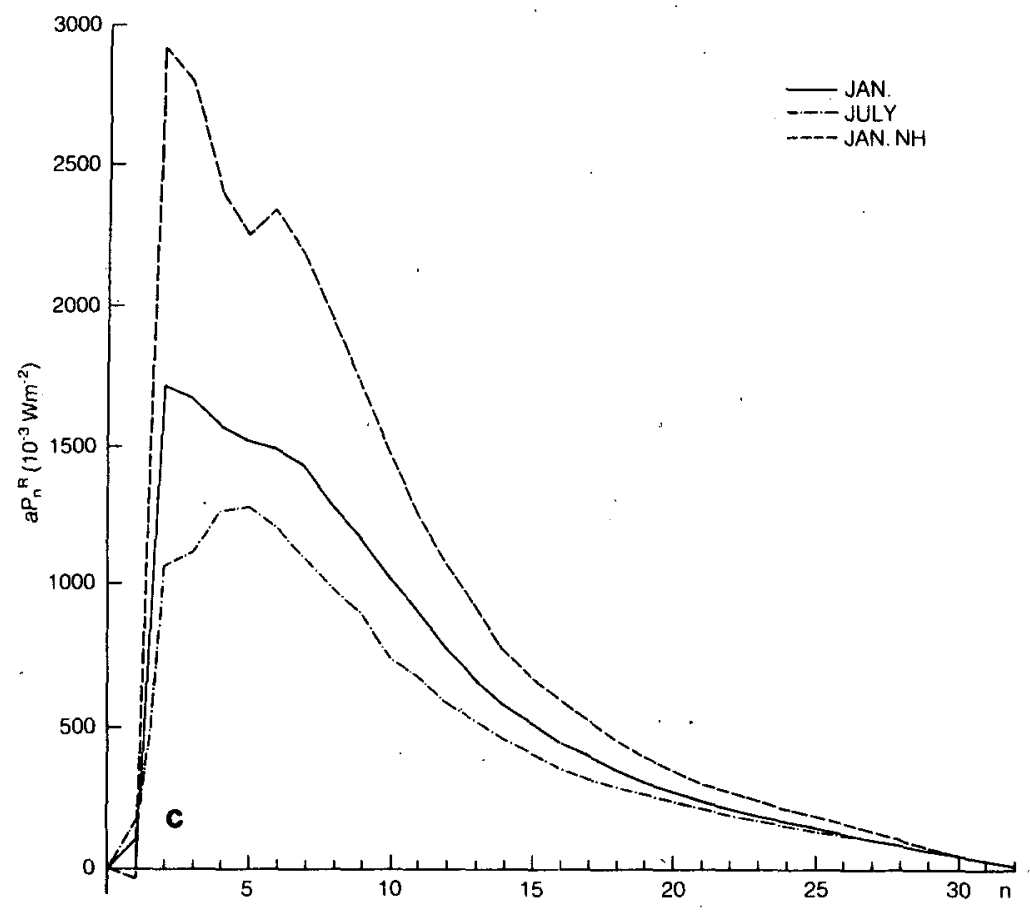

FIG. 10c. As in Fig. 10a but for available potential energy.

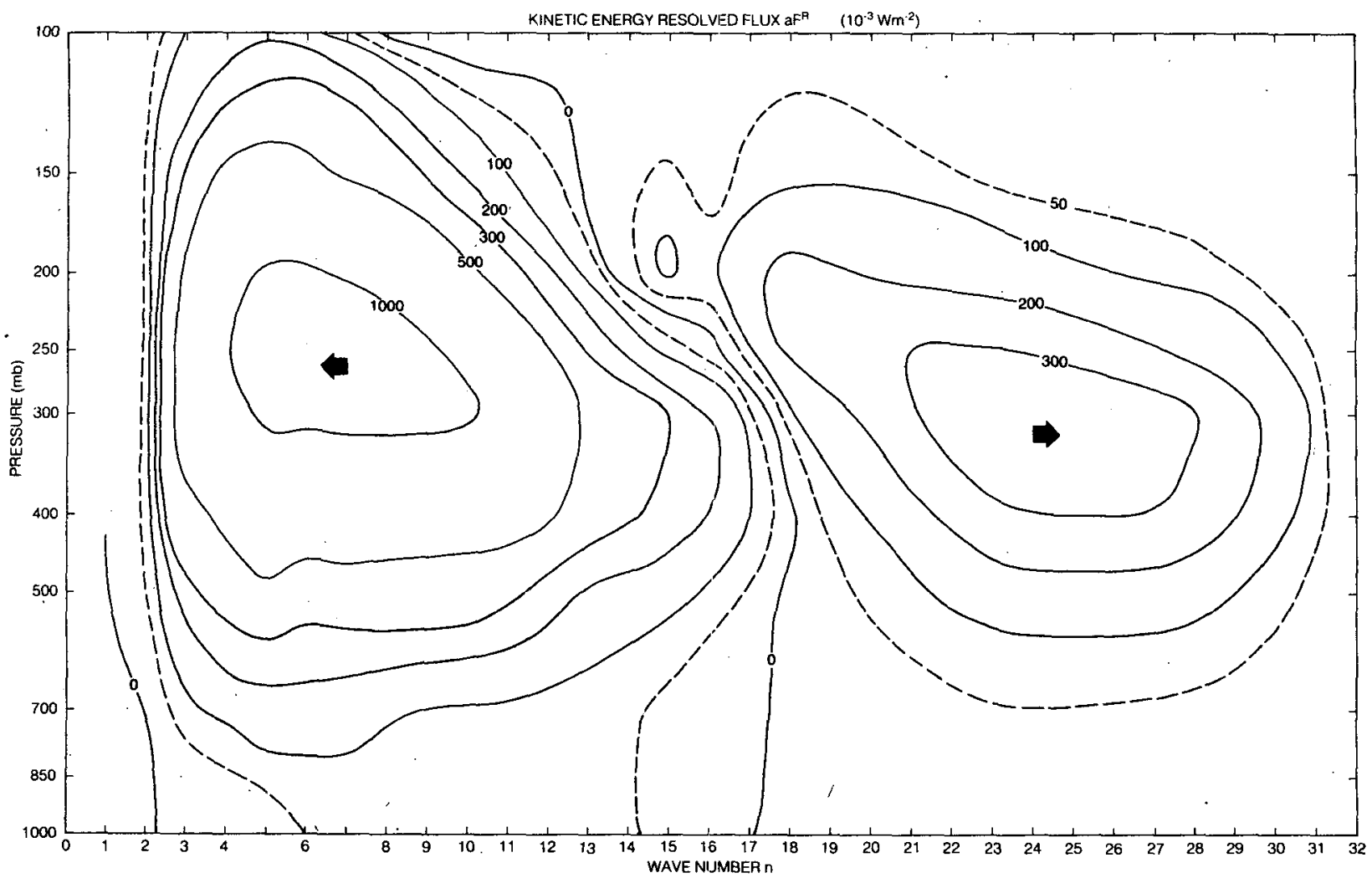

FiG. 11. Resolved part of the nonlinear kinetic energy flux term as a function of pressure. 
TABLE 1. Integrated and averaged values of resolved interaction term (units $10^{-3} \cdot \mathrm{W} \mathrm{m}^{-2}$ ).

\begin{tabular}{|c|c|c|c|c|}
\hline \multirow[b]{2}{*}{$n$} & \multicolumn{2}{|c|}{$I_{n}^{R}$} & \multicolumn{2}{|c|}{$M_{n}^{R}$} \\
\hline & January & July & January & July \\
\hline 1 & 1 & 0 & -93 & -151 \\
\hline 2 & 17 & 21 & -1627 & -923 \\
\hline 3 & 250 & 349 & 44 & -50 \\
\hline 4 & 60 & -12 & 105 & -146 \\
\hline 5 & 96 & 9 & 52 & -15 \\
\hline 6 & -37 & -6 & 26 & 70 \\
\hline 7 & 9 & -74 & 60 & 105 \\
\hline 8 & -22 & 23 & 139 & 119 \\
\hline 9 & -33 & -71 & 116 & 82 \\
\hline 10 & -24 & -29 & 146 & 153 \\
\hline 11 & -43 & -13 & 124 & 70 \\
\hline 12 & -43 & -31 & 131 & 98 \\
\hline 13 & -98 & -37 & 100 & 59 \\
\hline 14 & -24 & -16 & 91 & 67 \\
\hline 15 & -33 & -41 & 61 & 55 \\
\hline 16 & -29 & -48 & 71 & 42 \\
\hline 17 & -47 & -43 & 51 & 45 \\
\hline 18 & -48 & -34 & 52 & 29 \\
\hline 19 & -22 & -10 & 46 & 24 \\
\hline 20 & -11 & -17 & 27 & 26 \\
\hline 21 & -20 & -16 & 33 & 23 \\
\hline 22 & -15 & -10 & 26 & 26 \\
\hline 23 & -21 & -4 & 27 & 17 \\
\hline 24 & 6 & -1 & 24 & 18 \\
\hline 25 & -1 & 2 & 18 & 21 \\
\hline 26 & 7 & 10 & 22 & 16 \\
\hline 27 & 7 & 9 & 21 & 15 \\
\hline 28 & 15 & 14 & 20 & 26 \\
\hline 29 & 20 & 17 & 22 & 15 \\
\hline 30 & 24 & 15 & 20 & 25 \\
\hline 31 & 31 & 22 & 22 & 18 \\
\hline 32 & 27 & 20 & 23 & 21 \\
\hline
\end{tabular}

unresolved flux might be expected to maintain the value at a constant. These kinds of statements are, however, unproveable with the available data.

\section{f. The low-wavenumber regime}

The discussion of Section 2a suggested the possibility of a source-sink region at intermediate wavelengths connected by energy and enstrophy cascading turbulent subranges to a low-wavenumber sink of energy and a high-wavenumber sink of enstrophy. While it was argued above that the high-wavenumber regime is not inconsistent with such a general picture, the low-wavenumber regime certainly does not correspond to the energy-cascading turbulent subrange of simple theory. This region is dominated by the stationary component, the spectral slopes are not those of the simple theory, and isotropy and equipartition are notably lacking. There is a strong upscale flux of energy and a weak enstrophy flux but these cannot be interpreted within the simple turbulence picture. The manner in which this upscale energy flux is accomplished, in the low-wavenumber regime, by the transient and stationary components of the flow and the nature of the interaction between the components is an interesting area of potential study.

\section{g. Parameterization of the effects of unresolved scales}

A difficult and important problem associated with the limited resolution of atmospheric observations and models is the problem of understanding and parameterizing the effects of unresolved scales on those explicitly resolved. In the previous section, only the "resolved" interaction and flux terms were calculated.

In Section 3c an approach suggested by Leith (1971) and based on the presumption of the existence of an inertial subrange of two-dimensional turbulence was discussed. The high-wavenumber regime of the data possesses at least some aspects of the turbulent conditions on which Leith's parameterization is based. It is interesting to estimate the unresolved fluxes of enstrophy and energy from Eq. (19) in order to estimate the total spectral flux. The function $f(n)$ $n_{*}$ ) is shown in Fig. 12.

Fig. 13 displays the resolved energy and enstrophy fluxes $F_{n}^{R}, H_{n}^{R}$ together with the estimates of the total fluxes obtained by adding to them the estimated values of $F_{n}^{U}, H_{n}^{U}$ as obtained using (19). The resulting total energy flux estimate differs from the resolved flux by a not inconsiderable amount. In particular, in this case for $n_{*}=32$, it is interesting to note that the negative region of $f\left(n / n_{*}\right)$, although small in magnitude, multiplies the larger spectral amplitudes and so gives a relatively large effect at lower wavenumbers. The result is that upscale energy flux is now found at almost all wavenumbers.

The resulting energy and enstrophy fluxes may be compared with the requirements of zero energy and constant enstrophy flux for an inertial subrange. While the conditions are not exactly fulfilled it can be argued that the estimated net energy flux is quite close to zero for higher wavenumbers while the enstropy flux shows a tendency at least to adopt a constant value.

To perform these calculations a value of $\eta$ is required. The proper choice is not completely clear. It is not a very sensitive parameter however since it enters the formula as $\eta^{1 / 3}$. Ideally a "self-consistent" value should be chosen so that the resulting constant enstrophy cascade rate is just the value chosen. This can only be the case when the various assumptions under which (19) is obtained are satisfied. This is not the case here. In particular, the slope of the spectrum is shallower than -3 and this results in the enstrophy flux increasing with wavenumber rather than becoming flat. The value of $\eta$ chosen for these computations 


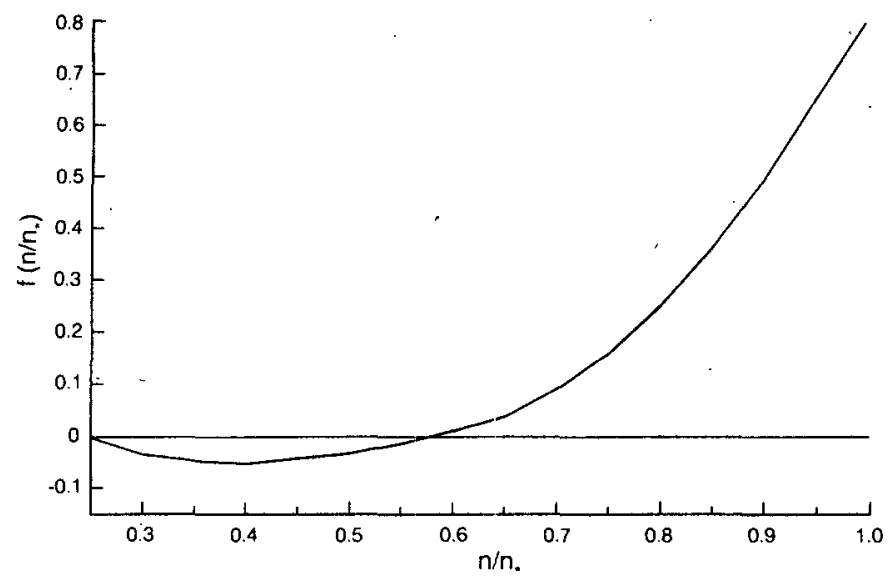

FIG. 12. Dissipative function in Eq. (19).
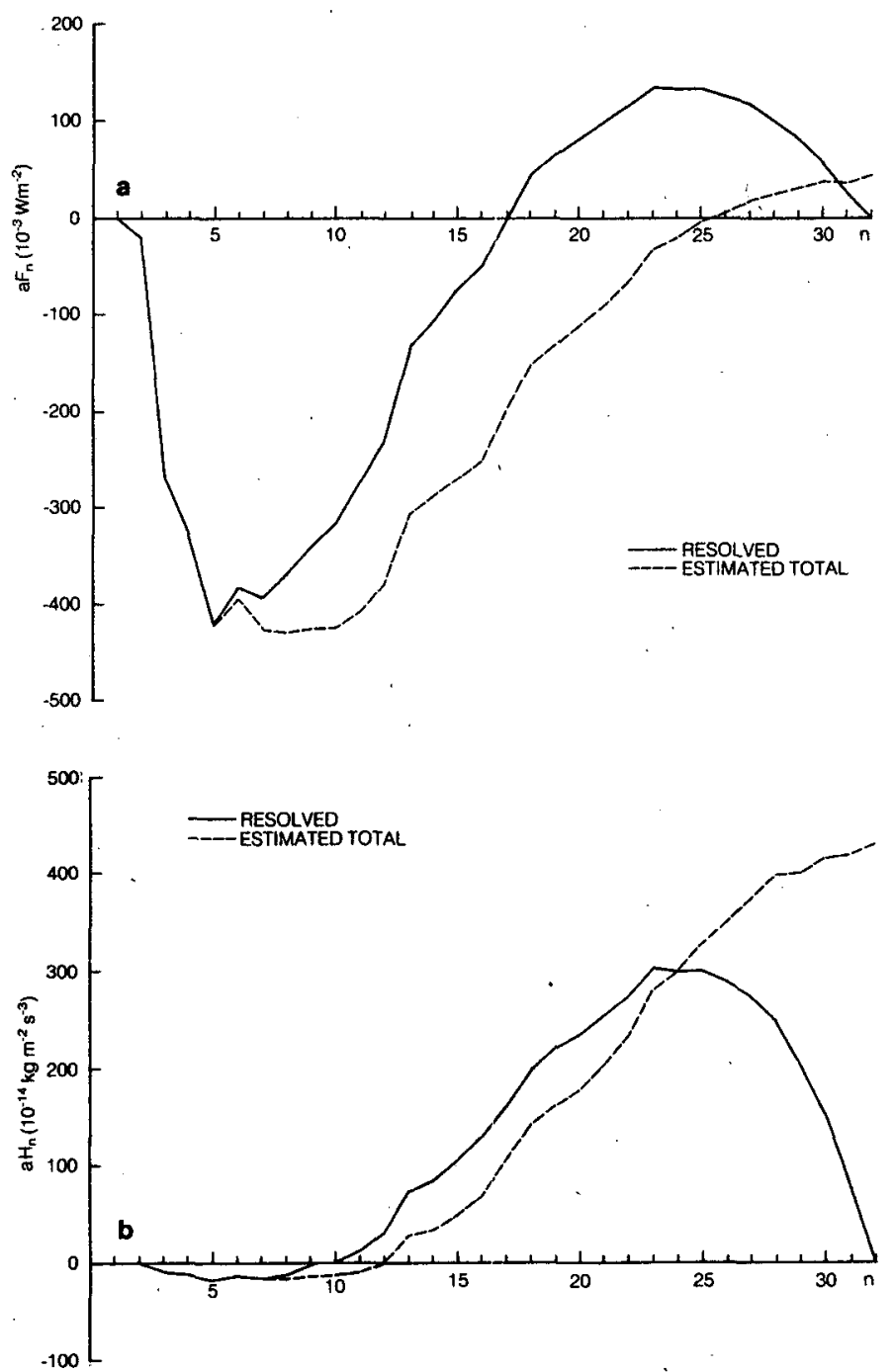

FIG. 13. Resolved and estimated total kinetic energy flux (a) and enstrophy (b). 
is $\eta=300 \times 10^{-18} \mathrm{~s}^{-3}$ corresponding to the maximum of the resolved flux.

This question of the parameterization of unresolved scales is an important practical one. Numerical models of the atmosphere, for instance, must include some such parameterization if they are to be integrated for long periods of time. If the fluxes of energy and enstrophy are known from high-resolution data or model output it is possible to calculate the values of $f_{n}$ for which the high-resolution fluxes can be recovered based on a lower resolution calculation and the parameterization of the unresolved scales. Thus if $I_{n}$ is known in some fashion it is possible to calculate $f_{n}$ from

$$
I_{n}=I_{n}^{R}-2 \eta^{1 / 3} f_{n} E_{n}
$$

This is independent of any particular assumptions about the nature of the flow. The function $f_{n}$ could also be a function of pressure for instance. Of course $f_{n}$ calculated in this way need not produce a universal function which is the answer to the parameterization problem. The shape of the function and its resemblance to the version obtained by Leith may throw some light on this problem however.

\section{Concluding remarks}

FGGE IIIa global data sets for January and July 1979 are used to calculate the spectra, interaction terms and fluxes of kinetic energy, enstrophy, and available potential energy in terms of the two-dimensional spherical harmonic wavenumber $n$.

The calculation clearly reveals two rather different regimes in wavenumber space. What is termed the high-wavenumber regime is dominated by the transient component of the flow and exhibits, at least approximately, several of the necessary conditions for homogeneous and isotropic flow on the sphere. These include the approximate independence of $\overline{\left|\psi_{n}^{m}\right|^{2}}$ on $m$ and the equipartition of energy between the two components of the kinetic energy and the available potential energy. The spectra in this region display a power law behavior with slopes somewhat shallower than -3 for energy and -1 for enstrophy. Slopes are steepest and energy densities largest in the upper troposphere. The spectra are remarkably unchanged between January and July. This region in wavenumber space is also associated with a strong flux of enstrophy and relatively weak flux of energy to higher wavenumbers.

The high wavenumber regime exhibits, in an approximate way, some of the features expected in an enstrophy-cascading inertial subrange. It is difficult to characterize the situation more precisely since only the "resolved" interactions and fluxes may be calculated.
In view of this behavior in the high-wavenumber regime, some consequences of the parameterization of the effects of unresolved scales on the resolved scales are considered. In particular the approach suggested by Leith (1971) is shown to give a plausible parameterization.

The low-wavenumber regime of the flow is quite different from the high-wavenumber regime which at least approximately exhibits behavior associated with simple models of turbulence. By contrast, the lowwavenumber regime is dominated by the stationary component of the flow and exhibits marked anisotropy as well as a lack of equipartition of energy. The spectra do not exhibit power law behavior and, in contrast with the case at high wavenumbers, show marked changes between January and July. This wavenumber regime displays a strong upscale flux of energy and a weak enstrophy flux.

The low-wavenumber region does not approximate any of the features of an energy-cascading inertial subrange except the upscale flux of energy. It acts as a sink of energy supplied from higher wavenumbers. The nature of the processes which maintain this stationary part of the flow and, in particular, the way in which both the transient and stationary parts of the flow act and interact to provide the upscale energy flux in this wavenumber region is an important area of future study.

Acknowledgments. We should like to thank Lynda Smith for her help with the figures and the manuscript. John Henderson was, as usual, very helpful with the computational aspects of the study.

\section{REFERENCES}

Baer, F., 1972: An alternate scale representation of atmospheric energy spectra. J. Atmos. Sci., 29, 649-664.

- 1974: Hemispheric spectral statistics of APE. J. Atmos. Sci., 31, 932-941.

- 1981: Three-dimensional scaling and structure of atmospheric energetics. J. Atmos. Sci., 38, 53-68.

Basdevant, C., B. Legras, R. Sadourny and M. Beland, 1981: A study of barotropic model flows: Intermittency, waves and predictability. J. Atmos. Sci., 38, 2305-2326.

Batchelor, G. K., 1953: The Theory of Homogeneous Turbulence. Cambridge University Press, $197 \mathrm{pp}$.

- 1969: Computation of the energy spectrum in homogeneous two-dimensional turbulence. Phys. Fluids, Suppl. II, 12, 233239.

Boer, G. J., 1983: Homogeneous and isotropic turbulence on the sphere. J. Atmos. Sci., 40, 154-163.

Bourke, W., 1972: An efficient one-level primitive equation spectral model. Mon. Wea. Rev., 100, 683-689.

Charney, J. G., 1971: Geostrophic turbulence. J. Atmos. Sci., 28, 1087-1095.

Chen, T.-C., and A. Wiin-Nielsen, 1978: Non-linear cascades of atmospheric energy and enstrophy in a two-dimensional spectral index. Tellus, 30, 313-322.

Fjørtøf, R., 1953: On the changes in the spectral distribution of kinetic energy for two-dimensional non-divergent flow. Tellus, 5, 225-230. 
Kolmogorov, A. N., 1941: The local structure of turbulence in incompressible viscous fluid for very large Reynolds numbers. C.R. Acad. Sci. URSS, 30, 301-305. [Dokl. Acad. Nauk. SSSR, 30, No. 4, 299-303.]

Kraichnan, R. H., 1967: Inertial subranges in two-dimensional turbulence. Phys. Fluids, 10, 1417-1423.

Kung, E. C., and W. E. Baker, 1975: Energy transformations in middle-latitude disturbances. Quart. J. Roy. Meteor. Soc., 101, 793-816.

Lambert, S. J., 1981: A diagnostic study of global energy and enstrophy fluxes and spectra. Tellus, 33, 411-414.

Leith, C. E., 1967: Diffusion approximation to inertial energy transfer in isotropic túrbulence. Phys. Fluids, 10, 1409-1416.

- , 1968: Diffusion approximation for two-dimensional turbulence. Phys. Fluids, 11, 671-673.

- 1971: Atmospheric predictability and two-dimensional turbulence. J. Atmos. Sci., 28, 145-161.
Lilly, D. K., 1972: Numerical simulation studies of two-dimensional turbulence. Geophys. Fluid Dyn., 3, 289-319; 4, 1-28.

McPherson, R. D., K. H. Bergman, R. E. Kistler, G. E. Rasch and D. S. Gordon, 1979: The NMC operational global data assimilation system. Mon. Wea. Rev., 107, 1445-1461.

Platzman, G. W., 1960: The spectral form of the vorticity equation. J. Meteor., 17, 635-644.

Rhines, P., 1975: Waves and turbulence on a $\beta$-plane. J. Fluid Mech., 69; 417-443.

- 1 1979: Geostrophic turbulence. Annual Review of Fluid Mechanics, Vol. 11, Annual Reviews, Inc., 401-441.

Tang, C-M., and S. A. Orszag, 1978: Two-dimensional turbulence on the surface of a sphere. J. Fluid Mech., 87, 305-319.

Tennekes, H., 1978: Turbulent flow in two and three dimensions. Bull. Amer. Meteor. Soc., 59, 22-28.

Wiin-Nielsen, A., 1972: A study of power laws in the atmospheric kinetic energy spectrum using spherical harmonic functions. Meteor. Ann., 6, 107-124. 